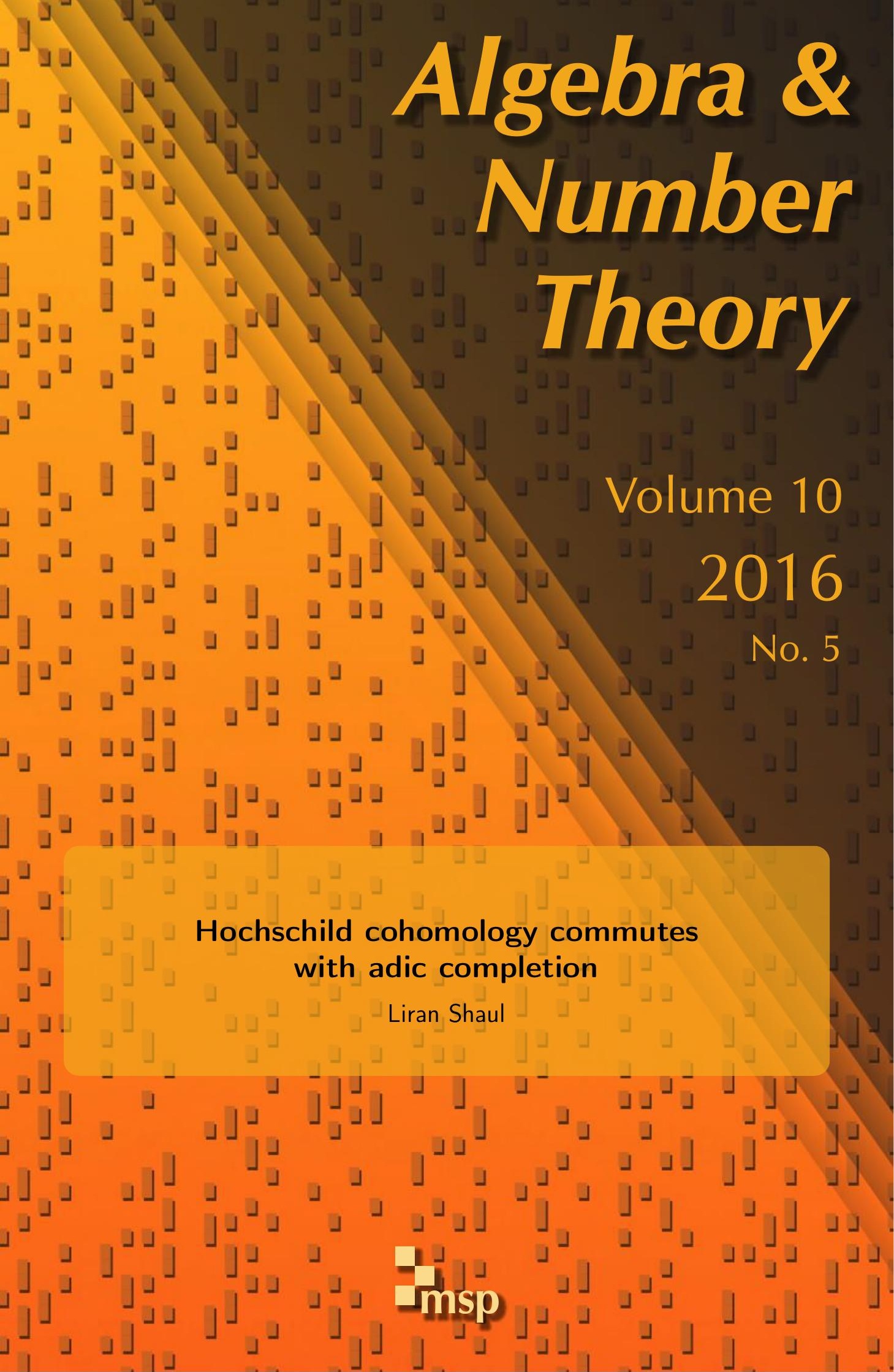




\title{
Hochschild cohomology commutes with adic completion
}

\author{
Liran Shaul
}

For a flat commutative $\mathbb{k}$-algebra $A$ such that the enveloping algebra $A \otimes_{\mathbb{k}} A$ is noetherian, given a finitely generated bimodule $M$, we show that the adic completion of the Hochschild cohomology module $\operatorname{HH}^{n}(A / \mathbb{k}, M)$ is naturally isomorphic to $\operatorname{HH}^{n}(\hat{A} / \mathbb{k}, \hat{M})$. To show this, we make a detailed study of derived completion as a functor $\mathrm{D}(\operatorname{Mod} A) \rightarrow \mathrm{D}(\operatorname{Mod} \hat{A})$ over a nonnoetherian ring $A$, prove a flat base change result for weakly proregular ideals, and prove that Hochschild cohomology and analytic Hochschild cohomology of complete noetherian local rings are isomorphic, answering a question of Buchweitz and Flenner. Our results make it possible for the first time to compute the Hochschild cohomology of $\mathbb{k} \llbracket t_{1}, \ldots, t_{n} \rrbracket$ over any noetherian ring $\mathbb{k}$, and open the door for a theory of Hochschild cohomology over formal schemes.

Introduction

1001

1. Preliminaries on completion, torsion, Hochschild cohomology 1004

2. Weak proregularity and flat base change 1007

3. The functors $R \hat{\Gamma}_{\mathfrak{a}}, \mathrm{L} \hat{\Lambda}_{\mathfrak{a}} \quad 1010$

4. Hochschild cohomology and derived completion 1018

5. Hochschild homology and derived torsion 1027

Acknowledgments 1027

References 1028

All rings in this paper are assumed to be commutative and unital.

\section{Introduction}

Hochschild cohomology [1945] has been the prominent cohomology theory for associative algebras since its introduction. In commutative algebra and algebraic geometry, its importance was first demonstrated by the celebrated theorem of

The author acknowledges the support of the European Union for ERC Grant No. 257004-HHNcdMir. MSC2010: primary 13D03; secondary 13J10, 14B15, 16E45, 13B35.

Keywords: Hochschild cohomology, adic completion. 
Hochschild, Kostant and Rosenberg. See [Ionescu 2001] for a survey of the use of Hochschild cohomology in commutative algebra.

The aim of this paper is to initiate the study of Hochschild cohomology in the category of adic rings. Adic rings, the affine pieces of the theory of formal schemes, are by definition commutative noetherian rings $A$ which are a-adically complete with respect to some ideal $\mathfrak{a} \subseteq A$. In the survey just cited, Ionescu states:

In our survey no results about Hochschild cohomology of a topological algebra w[ere] mentioned. This is because this kind of results is missing completely.

As far as we know, little has changed regarding this statement since then. One of the main difficulties in developing such a theory can be already observed in the most simple example of an adic ring: Let $\mathbb{k}$ be a field of characteristic 0 , and let $A=\mathbb{k} \llbracket t \rrbracket$. The construction of Hochschild cohomology involves the enveloping algebra of $A$. But even in this simple case, the enveloping algebra $\mathbb{k} \llbracket t \rrbracket \otimes_{\mathbb{k}} \mathbb{k} \llbracket t \rrbracket$ is a nonnoetherian ring of infinite Krull dimension, so it is very difficult to do homological algebra over it. Passing to the completion of this enveloping algebra, one obtains the much more manageable completed tensor product

$$
\mathbb{k} \llbracket t \rrbracket \hat{\otimes}_{\mathbb{k}} \mathbb{k} \llbracket t \rrbracket \cong \mathbb{k} \llbracket t_{1}, t_{2} \rrbracket .
$$

However, from a homological point of view, this step is highly nontrivial, as it involves the ring map from $\mathbb{k} \llbracket t \rrbracket \otimes_{\mathbb{k}} \mathbb{k} \llbracket t \rrbracket$ to its completion. Completions of nonnoetherian rings are in general poorly behaved (for instance, they need not be flat). In this paper we develop the homological tools needed to overcome this difficulty, and use them to study the Hochschild cohomology of such adic algebras.

Here is a more detailed description of the content of this paper. First, in Section 1 we review some preliminaries on Hochschild cohomology and about the derived torsion and derived completion functors. In particular, we recall the notion of a weakly proregular ideal in a commutative ring. Weak proregularity is the right condition in order for the derived torsion and derived completion functors to possess good behavior.

In Section 2, we prove that in most enveloping algebras of adic rings occurring in nature, the ideal of definition of the adic topology is weakly proregular. This result also has interesting implications in derived algebraic geometry of formal schemes. See Corollary 2.9.

Given a (not necessarily noetherian) ring $A$ and a weakly proregular ideal $\mathfrak{a} \subseteq A$, we study in Section 3 the derived functors of the functors

$$
\hat{\Gamma}_{\mathfrak{a}}(M):=\underline{\lim _{\longrightarrow}} \operatorname{Hom}_{A}\left(A / \mathfrak{a}^{n}, M\right): \operatorname{Mod} A \rightarrow \operatorname{Mod} \hat{A}
$$

and

$$
\hat{\Lambda}_{\mathfrak{a}}(M):=\lim A / \mathfrak{a}^{n} \otimes_{A} M: \operatorname{Mod} A \rightarrow \operatorname{Mod} \hat{A},
$$


where $\hat{A}$ is the $\mathfrak{a}$-adic completion of $A$. To cope with the possible lack of flatness of the completion map $A \rightarrow \hat{A}$, we use DG-homological algebra techniques. From the results of this section, we deduce the following generalized Greenlees-May duality:

Corollary 3.9. Let $A$ be a commutative ring, let $\mathfrak{a} \subseteq A$ be a weakly proregular ideal, and let $M, N \in \mathrm{D}(\operatorname{Mod} A)$. Then there are isomorphisms

$$
\mathrm{L} \hat{\Lambda}_{\mathfrak{a}}\left(\mathrm{R}_{\operatorname{Hom}_{A}}(M, N)\right) \cong \mathrm{R} \operatorname{Hom}_{A}\left(\mathrm{R} \hat{\Gamma}_{\mathfrak{a}}(M), N\right) \cong \operatorname{R}_{\operatorname{Hom}_{A}}\left(M, \operatorname{L} \hat{\Lambda}_{\mathfrak{a}}(N)\right)
$$

of functors

$$
\mathrm{D}(\operatorname{Mod} A) \times \mathrm{D}(\operatorname{Mod} A) \rightarrow \mathrm{D}(\operatorname{Mod} \hat{A}) .
$$

Using the results of Sections 2 and 3, the main results of this paper are obtained in Section 4. First, in Theorem 4.1 we provide formulas which describe the effect of applying the derived completion functor to the Hochschild cohomology complex of a not necessarily adic algebra. The next major result reduces the problem of computing the Hochschild cohomology of an adic algebra to a problem over noetherian rings:

Corollary 4.3. Let $\mathbb{k}$ be a commutative ring, and let $A$ be a flat noetherian $\mathbb{k}$ algebra. Assume $\mathfrak{a} \subseteq A$ is an ideal, such that $A$ is $\mathfrak{a}$-adically complete, and such that $A / \mathfrak{a}$ is essentially of finite type over $\mathbb{k}$. Let $I:=\mathfrak{a} \otimes_{\mathbb{k}} A+A \otimes_{\mathbb{k}} \mathfrak{a}$, and set $A \hat{\otimes}_{\mathbb{k}} A:=\Lambda_{I}\left(A \otimes_{\mathbb{k}} A\right)$. Then for any $M \in \operatorname{Mod} A \otimes_{\mathbb{k}} A$ which is I-adically complete (for example, any $\mathfrak{a}$-adically complete A-module, or more particularly, any finitely generated A-module), there is a functorial isomorphism

$$
\mathrm{R} \operatorname{Hom}_{A \otimes_{\mathbb{k}} A}(A, M) \cong \mathrm{R}_{\operatorname{Hom}_{A \hat{\otimes}_{\mathbb{k}} A}}(A, M)
$$

in $\mathrm{D}(\operatorname{Mod} A)$, and the ring $A \hat{\otimes}_{\mathbb{k}} A$ is noetherian.

Using the results of [Buchweitz and Flenner 2006], as a corollary of this result, we are able to prove in Corollary 4.5 that Hochschild cohomology and analytic Hochschild cohomology of complete noetherian local algebras coincide, answering a question of Buchweitz and Flenner. Finally, Section 4 ends with a theorem which proves the result mentioned in the title of the paper. More precisely, we show:

Theorem 4.13. Let $\mathbb{k}$ be a commutative ring, and let $A$ be a flat noetherian $\mathbb{k}$-algebra such that $A \otimes_{\mathbb{k}} A$ is noetherian. Let $\mathfrak{a} \subseteq A$ be an ideal, and let $M$ be a finitely generated $\left(A \otimes_{\mathbb{k}} A\right)$-module. Then for any $n \in \mathbb{N}$, there is a functorial isomorphism

$$
\Lambda_{\mathfrak{a}}\left(\operatorname{Ext}_{A \otimes_{\mathbb{k}} A}^{n}(A, M)\right) \cong \operatorname{Ext}_{\hat{A} \otimes_{\mathbb{k}} \hat{A}}^{n}(\hat{A}, \hat{M}) .
$$

If, moreover, either

(1) $\mathbb{k}$ is a field, or 
(2) A is projective over $\mathbb{k}$, a is a maximal ideal, and $M$ is a finitely generated A-module,

then there is also a functorial isomorphism

$$
\Lambda_{\mathfrak{a}}\left(\mathrm{HH}^{n}(A / \mathbb{k}, M)\right) \cong \operatorname{HH}^{n}(\hat{A} / \mathbb{k}, \hat{M}) .
$$

In the short and final Section 5, we briefly discuss analogous results for Hochschild homology.

Warning. Contrary to the convention in many papers in the field, unless stated otherwise, we do not assume that rings are noetherian.

\section{Preliminaries on completion, torsion and Hochschild cohomology}

Given a commutative ring $A$, we denote by $\operatorname{Mod} A$ the abelian category of $A$-modules, and by $\mathrm{D}(\operatorname{Mod} A)$ its (unbounded) derived category. If $A$ is noetherian, we will denote by $\mathrm{D}_{\mathrm{f}}(\operatorname{Mod} A)$ the triangulated subcategory made of complexes with finitely generated cohomologies. We will freely use resolutions of unbounded complexes, following [Spaltenstein 1988].

Completion and torsion. References for the material in this section are [Alonso Tarrío et al. 1997; 1999; Greenlees and May 1992; Porta et al. 2014b; 2015; Schenzel 2003; Simon 1990; Yekutieli 2011]. See [Porta et al. 2014b, Remark 7.14] for a brief discussion on the history of this material. Let $A$ be a commutative ring, and let $\mathfrak{a} \subseteq A$ be a finitely generated ideal. The $\mathfrak{a}$-torsion functor $\Gamma_{\mathfrak{a}}(-): \operatorname{Mod} A \rightarrow \operatorname{Mod} A$ is defined by

$$
\Gamma_{\mathfrak{a}}(M):=\lim _{\longrightarrow} \operatorname{Hom}_{A}\left(A / \mathfrak{a}^{n}, M\right) .
$$

This functor is a left exact additive functor. We denote its (total) right derived functor by $\mathrm{R} \Gamma_{\mathfrak{a}}: \mathrm{D}(\operatorname{Mod} A) \rightarrow \mathrm{D}(\operatorname{Mod} A)$. It is computed using K-injective resolutions. See [Brodmann and Sharp 2013] for a detailed study of the a-torsion functor and its derived functor in the noetherian case. More important in this paper is the a-adic completion functor, defined by

$$
\Lambda_{\mathfrak{a}}(-): \operatorname{Mod} A \rightarrow \operatorname{Mod} A, \quad \Lambda_{\mathfrak{a}}(M):=\lim _{\longleftarrow} A / \mathfrak{a}^{n} \otimes_{A} M .
$$

This functor is additive, but in general is neither left exact nor right exact (even when $A$ is noetherian; see [Yekutieli 2011, Example 3.20]). It does however preserve surjections. We denote by $\mathrm{L} \Lambda_{\mathfrak{a}}: \mathrm{D}(\operatorname{Mod} A) \rightarrow \mathrm{D}(\operatorname{Mod} A)$ its left derived functor. By [Alonso Tarrío et al. 1997, Section 1], it can be computed using K-flat resolutions. Both of the functors $\Gamma_{\mathfrak{a}}(-), \Lambda_{\mathfrak{a}}(-)$ are idempotent [Yekutieli 2011, Corollary 3.6].

For any ring $A$, the $A$-module $\Lambda_{\mathfrak{a}}(A)$ has the structure of a commutative $A$-algebra, called the completion of $A$. If $A$ is noetherian then $\Lambda_{\mathfrak{a}}(A)$ is flat over $A$, but if $A$ is not noetherian this does not always holds. For example, if $A$ is any countable 
ring which is not coherent, then the completion map $A[x] \rightarrow A \llbracket x \rrbracket$ is not flat [Stacks 2005-, Tag 0AL8]. The ring $\Lambda_{\mathfrak{a}}(A)$ is noetherian if and only if the ring $A / \mathfrak{a}$ is noetherian [Stacks 2005-, Tag $05 \mathrm{GH}$ ]. If $A$ is noetherian and $M$ is a finitely generated $A$-module, then there is an isomorphism of functors $\Lambda_{\mathfrak{a}}(M) \cong$ $\Lambda_{\mathfrak{a}}(A) \otimes_{A} M$, so in particular in that case, $\Lambda_{\mathfrak{a}}(-)$ is exact on the category of finitely generated $A$-modules [Stacks $2005-$, Tag $00 \mathrm{MB}$ ]. We will sometimes denote by $\hat{A}$ the $A$-algebra $\Lambda_{\mathfrak{a}}(A)$ and by $\hat{M}$ the $A$-module $\Lambda_{\mathfrak{a}}(M)$. For any ring $A$, the $A$-modules $\Gamma_{\mathfrak{a}}(M)$ and $\Lambda_{\mathfrak{a}}(M)$ carry naturally the structure of $\hat{A}$-modules, and so one may view the $\mathfrak{a}$-torsion and $\mathfrak{a}$-completion functors as functors $\operatorname{Mod} A \rightarrow \operatorname{Mod} \hat{A}$. Section 3 is dedicated to a study of the functors obtained from this observation.

Given a ring $A$, and an element $\mathfrak{a} \in A$, the infinite dual Koszul complex associated to it is

$$
\mathrm{K}_{\infty}^{\vee}(A ;(a)):=\left(\cdots \rightarrow 0 \rightarrow A \rightarrow A\left[a^{-1}\right] \rightarrow 0 \rightarrow \cdots\right)
$$

concentrated in degrees 0,1 . If $\left(a_{1}, \ldots, a_{n}\right)$ is a finite sequence of elements in $A$, then the infinite dual Koszul complex associated to it is

$$
\mathrm{K}_{\infty}^{\vee}\left(A ;\left(a_{1}, \ldots, a_{n}\right)\right):=\mathrm{K}_{\infty}^{\vee}\left(A ;\left(a_{1}\right)\right) \otimes_{A} \cdots \otimes_{A} \mathrm{~K}_{\infty}^{\vee}\left(A ;\left(a_{n}\right)\right) .
$$

It is a bounded complex of flat $A$-modules. Given an ideal $\mathfrak{a} \subseteq A$, and a finite sequence $\boldsymbol{a}$ of elements of $A$ that generate $\mathfrak{a}$, by [Porta et al. 2014b, Corollary 4.26], there is a morphism of functors

$$
\mathrm{R} \Gamma_{\mathfrak{a}}(-) \rightarrow \mathrm{K}_{\infty}^{\vee}(A ; \boldsymbol{a}) \otimes_{A}-.
$$

The sequence $\boldsymbol{a}$ is called weakly proregular if this morphism is an isomorphism of functors. This notion is actually independent of $\boldsymbol{a}$, and depends only on the ideal $\mathfrak{a}$ generated by it [Schenzel 2003, Lemma 3.3]. Hence, we say a finitely generated ideal $\mathfrak{a}$ is weakly proregular if some (or, equivalently, any) finite sequence that generates it is weakly proregular. In a noetherian ring, any ideal and any finite sequence are weakly proregular, but there are examples of finitely generated (even principal) ideals in nonnoetherian rings which are not weakly proregular.

Given a ring $A$ and a finite sequence $\boldsymbol{a}$ of elements of $A$, the infinite dual Koszul complex has an explicit free resolution, called the telescope complex and denoted by $\operatorname{Tel}(A ; \boldsymbol{a})$. This resolution is a bounded complex of countably generated free $A$-modules [Porta et al. 2014b, Lemma 5.7]. In particular, if the ideal $\mathfrak{a}$ generated by $\boldsymbol{a}$ is weakly proregular, then there is also an isomorphism of functors

$$
\mathrm{R} \Gamma_{\mathfrak{a}}(-) \cong \operatorname{Tel}(A ; \boldsymbol{a}) \otimes_{A}-.
$$

Moreover, in this case, by [Porta et al. 2014b, Corollary 5.25], there is also an isomorphism of functors

$$
\mathrm{L} \Lambda_{\mathfrak{a}}(-) \cong \operatorname{Hom}_{A}(\operatorname{Tel}(A ; \boldsymbol{a}),-) .
$$


It follows that if $A$ is a commutative ring, and $\mathfrak{a}$ is a weakly proregular ideal, then both of the functors $R \Gamma_{\mathfrak{a}}, \mathrm{L} \Lambda_{\mathfrak{a}}$ have finite cohomological dimension, and there is a bifunctorial isomorphism, the Greenlees-May duality,

$$
\mathrm{R} \operatorname{Hom}_{A}\left(\mathrm{R} \Gamma_{\mathfrak{a}}(M), N\right) \cong \mathrm{R} \operatorname{Hom}_{A}\left(M, \mathrm{~L} \Lambda_{\mathfrak{a}}(N)\right)
$$

for any $M, N \in \mathrm{D}(\operatorname{Mod} A)$.

Both the infinite dual Koszul complex and the telescope complex enjoy the following base change property: if $A$ is a ring, $\boldsymbol{a}$ is a finite sequence of elements in $A$, $A \rightarrow B$ is a ring map, and $\boldsymbol{b}$ is the image of $\boldsymbol{a}$ under this map, then there are isomorphisms

$$
\mathrm{K}_{\infty}^{\vee}(A ; \boldsymbol{a}) \otimes_{A} B \cong \mathrm{K}_{\infty}^{\vee}(B ; \boldsymbol{b}), \quad \operatorname{Tel}(A ; \boldsymbol{a}) \otimes_{A} B \cong \operatorname{Tel}(B ; \boldsymbol{b})
$$

of complexes of $B$-modules.

For any complex $M \in \mathrm{D}(\operatorname{Mod} A)$, there are canonical maps

$$
\mathrm{R} \Gamma_{\mathfrak{a}}(M) \rightarrow M,
$$

and

$$
M \rightarrow \mathrm{L} \Lambda_{\mathfrak{a}}(M) .
$$

The complex $M$ is called cohomologically a-torsion (resp. cohomologically $\mathfrak{a}$ adically complete) if the map (1.1) (resp. (1.2)) is an isomorphism. If $\mathfrak{a}$ is weakly proregular then the functors $\mathrm{R} \Gamma_{\mathfrak{a}}$ and $\mathrm{L} \Lambda_{\mathfrak{a}}$ are idempotent [Porta et al. 2014b, Corollary 4.30, Proposition 7.10] and it follows that in this case the collection of all cohomologically a-torsion (resp. cohomologically $\mathfrak{a}$-adically complete) complexes is a triangulated subcategory of $\mathrm{D}(\operatorname{Mod} A)$ which is equal to the essential image of the functor $\mathrm{R} \Gamma_{\mathfrak{a}}\left(\operatorname{resp} . \mathrm{L} \Lambda_{\mathfrak{a}}\right)$. Moreover, by [Porta et al. 2014b, Theorem 7.11], in this case these categories are equivalent (the Matlis-Greenlees-May equivalence).

Hochschild cohomology. Let $\mathbb{k}$ be a commutative ring, and let $A$ be a commutative k-algebra. We let

$$
A^{\otimes_{k}^{n}}:=\underbrace{A \otimes_{\mathbb{k}} \cdots \otimes_{\mathbb{k}} A}_{n},
$$

and denote by $\mathcal{B}$ the bar resolution

$$
\cdots \rightarrow A^{\otimes_{k}^{n}} \rightarrow \cdots \rightarrow A^{\otimes_{k}^{2}} \rightarrow A \rightarrow 0 .
$$

Given an $A$-bimodule $M$, the $n$-th Hochschild cohomology module of $A$ over $\mathbb{k}$ with coefficients in $M$ is given by

$$
\operatorname{HH}^{n}(A / \mathbb{k}, M):=H^{n} \operatorname{Hom}_{A \otimes_{\mathbb{k}} A}(\mathcal{B}, M) .
$$

See [Cartan and Eilenberg 1956, Chapter IX], [Loday 1998, Chapter 1] and [Weibel 1994, Chapter 9] for more details on this classical construction. If $A$ is projective 
(resp. flat) over $\mathbb{k}$, then $\mathcal{B}$ is a projective (resp. flat) resolution of $A$ over the enveloping algebra $A \otimes_{\mathbb{k}} A$. Hence, in the projective case, the natural map

$$
\mathrm{HH}^{n}(A / \mathbb{k}, M) \rightarrow \operatorname{Ext}_{A \otimes_{\mathbb{k}} A}^{n}(A, M)
$$

is an isomorphism. When $A$ is only flat over $\mathbb{k}$, but not necessarily projective, this map might fail in general to be an isomorphism. Nevertheless, the modules on the right-hand side are interesting on their own, and are sometimes referred to in the literature as the derived Hochschild (or Shukla) cohomology modules of $A$ over $\mathbb{k}$. In this paper we will focus mostly on these modules ${ }^{1}$ and, a bit more generally, on the complex $\mathrm{RHom}_{A \otimes_{\mathbb{k}} A}(A, M)$. Somewhat imprecisely, we will refer to

$$
\mathrm{R} \operatorname{Hom}_{A \otimes_{k} A}(A, M)
$$

as the Hochschild complex of $A$ with coefficients in $M$ even when $A$ is only flat over $\mathbb{k}$. We will however use the notation $\operatorname{HH}^{n}(A / \mathbb{k}, M)$ to denote only the classical Hochschild cohomology modules.

\section{Weak proregularity and flat base change}

Let $\mathbb{k}$ be a base commutative ring, and let $A, B$ be two flat $\mathbb{k}$-algebras. Assume that $A$ and $B$ are equipped with adic topologies, generated by finitely generated ideals $\mathfrak{a} \subseteq A$ and $\mathfrak{b} \subseteq B$. In that case, the tensor product $A \otimes_{\mathbb{k}} B$ is also naturally equipped with an adic topology. It is generated by the finitely generated ideal $\mathfrak{a} \otimes_{\mathbb{k}} B+A \otimes_{\mathbb{k}} \mathfrak{b} \subseteq A \otimes_{\mathbb{k}} B$. The aim of this section is to discuss the question of when this ideal is weakly proregular. We allow $A$ to be different from $B$, although we will only use the case $A=B$ in the rest of the paper.

Recall that a ring $\mathbb{k}$ is called absolutely flat (or Von Neumann regular) if every $\mathbb{k}$-module is flat. Over such rings, the above question is easy:

Proposition 2.1. Let $\mathbb{k}$ be an absolutely flat ring. Let $A, B$ be two $\mathbb{k}$-algebras, and let $\mathfrak{a} \subseteq A$ and $\mathfrak{b} \subseteq B$ be weakly proregular ideals. Then the ideal

$$
\mathfrak{a} \otimes_{\mathbb{k}} B+A \otimes_{\mathbb{k}} \mathfrak{b} \subseteq A \otimes_{\mathbb{k}} B
$$

is weakly proregular.

Proof. In the case where $\mathbb{k}$ is a field, and $A$ and $B$ are noetherian and complete with respect to the adic topology, this is shown in [Porta et al. 2014b, Example 4.35], and the proof there remains true under the above assumptions.

Remark 2.2. Assume $A$ is a ring, $\mathfrak{a} \subseteq A$ is a weakly proregular ideal, $B$ is a flat $A$-algebra, and $\mathfrak{b}=\mathfrak{a} \cdot B$. Then by [Alonso Tarrío et al. 1997, Example 3.0(B)], the ideal $\mathfrak{b}$ is also weakly proregular.

\footnotetext{
${ }^{1}$ See, however, Corollary 4.5 and Theorem 4.13 where even in the possible absence of projectivity we will discuss classical Hochschild cohomology.
} 
Recall that if $A$ is a noetherian ring, with $\mathfrak{a} \subseteq A$ an ideal, and if $I$ is an injective $A$-module, then $\Gamma_{\mathfrak{a}}(I)$ is also an injective $A$-module (for example, by [Hartshorne 1977, Lemma 3.2]). We now state and prove a weaker form of this fact in the case when $A$ is not necessarily noetherian, but $\Lambda_{\mathfrak{a}}(A)$ is.

If $A$ is a ring, with $\mathfrak{a} \subseteq A$ a finitely generated ideal, and if $M$ is an $A$-module, then $M$ is called $\mathfrak{a}$-flasque if, for each $k>0$, we have $\mathrm{H}_{\mathfrak{a}}^{k}(M)=0$, where $\mathrm{H}_{\mathfrak{a}}^{k}(M):=$ $\mathrm{H}^{k}\left(\mathrm{R} \Gamma_{\mathfrak{a}}(M)\right)$. Any injective module is $\mathfrak{a}$-flasque. If $M$ is $\mathfrak{a}$-flasque, then the canonical morphism $\Gamma_{\mathfrak{a}}(M) \rightarrow \mathrm{R} \Gamma_{\mathfrak{a}}(M)$ is an isomorphism. By [Brodmann and Sharp 2013, Theorem 3.4.10], the direct limit of $\mathfrak{a}$-flasque modules is $\mathfrak{a}$-flasque.

Lemma 2.3. Let $A$ be a ring, and let $\mathfrak{a}, \mathfrak{b} \subseteq A$ be two finitely generated ideals. Suppose that the ring $\hat{A}=\Lambda_{\mathfrak{a}}(A)$ is noetherian. Let $\hat{\mathfrak{b}}=\mathfrak{b} \hat{A}$. Then for any injective A-module I, the $\hat{A}$-module $\hat{\Gamma}_{\mathfrak{a}} I$ is $\hat{\mathfrak{b}}$-flasque.

Proof. Let $A_{j}=A / \mathfrak{a}^{j+1}$. Since $\mathfrak{a}$ is finitely generated, there is an isomorphism $A_{j} \cong \hat{A} /(\mathfrak{a} \hat{A})^{j+1}$. Note that, by assumption, $A_{j}$ is noetherian. Let $\hat{\mathfrak{b}}_{j}$ be the image of $\hat{\mathfrak{b}}$ in $A_{j}$. Let $I_{j}=\operatorname{Hom}_{A}\left(A_{j}, I\right)$. Then $\hat{\Gamma}_{\mathfrak{a}} I=\varliminf_{\longrightarrow} I_{j}$, so it is enough to show that $I_{j}$ is $\hat{\mathfrak{b}}$-flasque. Note also that $I_{j}$ is an injective $\overrightarrow{A_{j}}$-module. Let $k>0$, let $\hat{\boldsymbol{b}}$ be a finite sequence generating $\hat{\mathfrak{b}}$, and let $\boldsymbol{b}_{j}$ be its image in $A_{j}$. Since $\hat{A}$ is noetherian, $\hat{\mathfrak{b}}$ is weakly proregular, so that

$$
H_{\hat{\mathfrak{b}}}^{k}\left(I_{j}\right) \cong H^{k}\left(\mathrm{~K}_{\infty}^{\vee}(\hat{A} ; \hat{\boldsymbol{b}}) \otimes_{\hat{A}} I_{j}\right) \cong H^{k}\left(\mathrm{~K}_{\infty}^{\vee}\left(A_{j} ; \boldsymbol{b}_{j}\right) \otimes_{A_{j}} I_{j}\right) \cong H_{\hat{\mathfrak{b}}_{j}}^{k}\left(I_{j}\right),
$$

where the last isomorphism follows from the fact that $A_{j}$ is noetherian, so that $\hat{\mathfrak{b}}_{j}$ is weakly proregular. Since $I_{j}$ is injective over $A_{j}$, it follows that $H_{\hat{\mathfrak{b}}_{j}}^{k}\left(I_{j}\right)=0$ for all $k>0$, which proves the claim.

Proposition 2.4. Let $A$ be a commutative ring, and let $\mathfrak{a} \subseteq A$ be a weakly proregular ideal such that $\Lambda_{\mathfrak{a}}(A)$ is noetherian. Let $\mathfrak{b} \subseteq A$ be an ideal containing $\mathfrak{a}$. Then $\mathfrak{b}$ is also weakly proregular.

Proof. We keep the notation of Lemma 2.3. It is clear that $A / \mathfrak{b}$ is noetherian. Let $\boldsymbol{a}$ be a finite sequence generating $\mathfrak{a}$, and let $\boldsymbol{b}$ be a finite sequence generating $\mathfrak{b}$. Let $I$ be an injective $A$-module. By [Schenzel 2003, Theorem 1.1], it is enough to show that

$$
H^{k}\left(\operatorname{Tel}(A ; \boldsymbol{b}) \otimes_{A} I\right)=0
$$

for all $k \neq 0$.

Since $\mathfrak{a} \subseteq \mathfrak{b}$, the ideal generated by the concatenated sequence $(\boldsymbol{a}, \boldsymbol{b})$ is equal to the ideal generated by $\boldsymbol{b}$, so there is a homotopy equivalence $\operatorname{Tel}(A ;(\boldsymbol{a}, \boldsymbol{b})) \cong \operatorname{Tel}(A ; \boldsymbol{b})$. Hence, there is an isomorphism

$$
\operatorname{Tel}(A ; \boldsymbol{b}) \otimes_{A} I \cong \operatorname{Tel}(A ;(\boldsymbol{a}, \boldsymbol{b})) \otimes_{A} I \cong \operatorname{Tel}(A ; \boldsymbol{b}) \otimes_{A} \operatorname{Tel}(A ; \boldsymbol{a}) \otimes_{A} I
$$


in $\mathrm{D}(\operatorname{Mod} A)$. Since $\boldsymbol{a}$ is a weakly proregular sequence, $I$ is an injective $A$-module, and $\operatorname{Tel}(A ; \boldsymbol{b})$ is a bounded complex of flat modules, the latter is isomorphic in $\mathrm{D}(\operatorname{Mod} A)$ to

$$
\operatorname{Tel}(A ; \boldsymbol{b}) \otimes_{A} \Gamma_{\mathfrak{a}} I .
$$

Thus, it is enough to show that all the cohomologies (except the zeroth) of the complex of $A$-modules $\operatorname{Tel}(A ; \boldsymbol{b}) \otimes_{A} \Gamma_{\mathfrak{a}} I$ vanish. Let

$$
\operatorname{Rest}_{\hat{A} / A}: \mathrm{D}(\operatorname{Mod} \hat{A}) \rightarrow \mathrm{D}(\operatorname{Mod} A)
$$

be the forgetful functor, and let $\hat{b}$ be the image of the sequence $\boldsymbol{b}$ in $\hat{A}$. Consider the complex

$$
\operatorname{Tel}(\hat{A} ; \hat{b}) \otimes_{\hat{A}} \hat{\Gamma}_{\mathfrak{a}} I \in \mathrm{D}(\operatorname{Mod} \hat{A}) .
$$

We claim that

$$
\operatorname{Rest}_{\hat{A} / A}\left(\operatorname{Tel}(\hat{A} ; \hat{\boldsymbol{b}}) \otimes_{\hat{A}} \hat{\Gamma}_{\mathfrak{a}} I\right)=\operatorname{Tel}(A ; \boldsymbol{b}) \otimes_{A} \Gamma_{\mathfrak{a}} I .
$$

Indeed, by the base change property of the telescope complex, we have an isomorphism

$$
\operatorname{Tel}(\hat{A} ; \hat{\boldsymbol{b}}) \otimes_{\hat{A}} \hat{\Gamma}_{\mathfrak{a}} I \cong \operatorname{Tel}(A ; \boldsymbol{b}) \otimes_{A} \hat{\Gamma}_{\mathfrak{a}} I
$$

of complexes in $\mathrm{D}(\operatorname{Mod} \hat{A})$. So using the fact that

$$
\operatorname{Rest}_{\hat{A} / A}\left(\hat{\Gamma}_{\mathfrak{a}} I\right)=\Gamma_{\mathfrak{a}} I
$$

we obtain (2.5). Since for a complex $M \in \mathrm{D}(\operatorname{Mod} \hat{A})$ we have

$$
H^{k}(M)=0 \quad \text { if and only if } \quad H^{k}\left(\operatorname{Rest}_{\hat{A} / A}(M)\right)=0,
$$

it is enough to show that $H^{k}\left(\operatorname{Tel}(\hat{A} ; \hat{\boldsymbol{b}}) \otimes_{\hat{A}} \hat{\Gamma}_{\mathfrak{a}} I\right)=0$ for all $k \neq 0$. By weak proregularity of the sequence $\hat{b}$, there is an isomorphism

$$
\operatorname{Tel}(\hat{A} ; \hat{\boldsymbol{b}}) \otimes_{\hat{A}} \hat{\Gamma}_{\mathfrak{a}} I \cong \mathrm{R} \Gamma_{\hat{\mathfrak{b}}} \hat{\Gamma}_{\mathfrak{a}} I
$$

in $\mathrm{D}(\operatorname{Mod} \hat{A})$. By Lemma 2.3 , this is isomorphic in $\mathrm{D}(\operatorname{Mod} \hat{A})$ to $\Gamma_{\hat{\mathfrak{b}}} \hat{\Gamma}_{\mathfrak{a}} I$. Since this complex is clearly concentrated in degree zero, it follows that all of its cohomologies except the zeroth vanish, which proves the result.

Here is the main result of this section:

Theorem 2.6. Let $\mathbb{k}$ be a commutative ring, let $A$ be a flat noetherian $\mathbb{k}$-algebra, and let $\mathfrak{a} \subseteq A$ be an ideal such that $A / \mathfrak{a}$ is essentially of finite type over $\mathbb{k}$. Let $B$ be a flat noetherian $\mathbb{k}$-algebra, and let $\mathfrak{b} \subseteq B$ be an ideal. Then the ideal

$$
I:=\mathfrak{a} \otimes_{\mathbb{k}} B+A \otimes_{\mathbb{k}} \mathfrak{b} \subseteq A \otimes_{\mathbb{k}} B
$$

is weakly proregular. 
Proof. According to Remark 2.2, the ideal $I_{1}:=\mathfrak{a} \otimes_{\mathbb{k}} B \subseteq A \otimes_{\mathbb{k}} B$ is weakly proregular. Since $B$ is flat over $\mathbb{k}$, we have $\left(A \otimes_{\mathbb{k}} B\right) / I_{1} \cong A / \mathfrak{a} \otimes_{\mathbb{k}} B$, and as $A / \mathfrak{a}$ is essentially of finite type over $\mathbb{k}$, it follows that $\left(A \otimes_{\mathbb{k}} B\right) / I_{1}$ is noetherian. Hence, $\Lambda_{I_{1}}\left(A \otimes_{\mathbb{k}} B\right)$ is also noetherian. Since $I_{1} \subseteq I$, the result follows from Proposition 2.4.

Remark 2.7. The assumption that $A$ is noetherian in the above result can be relaxed: it is enough to assume that $\mathfrak{a}$ is weakly proregular. It is an open problem to the author if the above result remains true without the assumption that $A / \mathfrak{a}$ is essentially of finite type over $\mathbb{k}$ (as in Proposition 2.1).

Remark 2.8. Let $\mathbb{k}$ be a commutative ring, and let $A, B$ be two commutative noetherian $\mathbb{k}$-algebras which are adically complete with respect to ideals $\mathfrak{a} \subseteq A$, $\mathfrak{b} \subseteq B$. In this situation, Grothendieck and Dieudonné [1960, Section 10.7] defined the fiber product of the two affine formal schemes $\operatorname{Spf} A, \operatorname{Spf} B$ over $\mathbb{k}$ to be the formal spectrum of the ring $\Lambda_{\mathfrak{a} \otimes_{\mathfrak{k}}} B+A \otimes_{\mathfrak{k}} \mathfrak{b}\left(A \otimes_{\mathbb{k}} B\right)$.

Now, we switch to the point of view of derived algebraic geometry, and assume that $A$ and $B$ are flat over $\mathbb{k}$. Forgetting the adic structure on $A, B$, the flatness assumption ensures that, in this situation, the usual fiber product $\operatorname{Spec}\left(A \otimes_{\mathbb{k}} B\right)$ of the schemes $\operatorname{Spec}(A)$ and $\operatorname{Spec}(B)$ coincides with their derived fiber product. Returning to the adic situation, Lurie defined [2011, Section 4.2] a notion of a derived completion of an $\left(E_{\infty}\right)$ ring, and showed [2011, Section 4.3] that if the ring is noetherian then its derived completion coincides with its ordinary completion. Recently, using our [Porta et al. 2014a, Theorem 4.2], it was shown in [Braunling et al. 2015, Proposition 5.4] that if $R$ is any commutative ring, and if $I \subseteq R$ is a weakly proregular ideal, then the derived $I$-completion of $R$ coincides with its ordinary $I$-adic completion. Hence, the results of this section imply the following:

Corollary 2.9. Let $\mathbb{k}$ be a commutative ring, and let $A, B$ be noetherian flat $\mathbb{k}$-algebras which are adically complete with respect to ideals $\mathfrak{a} \subseteq A, \mathfrak{b} \subseteq B$. Assume further that either $\mathbb{k}$ is an absolutely flat ring (e.g., a field) or that $A / \mathfrak{a}$ is essentially of finite type over $\mathbb{k}$. Then the derived fiber product of the formal schemes Spf $A$, Spf $B$ over $\mathbb{k}$ is equal to the formal spectrum of $\Lambda_{\mathfrak{a} \otimes_{\mathbb{k}} B+A \otimes_{\mathbb{k}} \mathfrak{b}}\left(A \otimes_{\mathbb{k}} B\right)$.

\section{The functors $R \hat{\Gamma}_{\mathfrak{a}}, \mathbf{L} \hat{\Lambda}_{\mathfrak{a}}$}

Let $A$ be a commutative ring, let $\mathfrak{a} \subseteq A$ be a finitely generated ideal, and let $\hat{A}$ be the a-adic completion of $A$. For any $A$-module $M$, the $A$-modules $\Gamma_{\mathfrak{a}}(M)$ and $\Lambda_{\mathfrak{a}}(M)$ carry naturally $\hat{A}$-module structures, and one obtains additive functors $\hat{\Gamma}_{\mathfrak{a}}, \hat{\Lambda}_{\mathfrak{a}}: \operatorname{Mod} A \rightarrow \operatorname{Mod} \hat{A}$, defined by the same formulas as $\Gamma_{\mathfrak{a}}$ and $\Lambda_{\mathfrak{a}}$. These functors have derived functors $\mathrm{R} \hat{\Gamma}_{\mathfrak{a}}, \mathrm{L} \hat{\Lambda}_{\mathfrak{a}}: \mathrm{D}(\operatorname{Mod} A) \rightarrow \mathrm{D}(\operatorname{Mod} \hat{A})$, calculated using K-injective and $\mathrm{K}$-flat resolutions, respectively. This section is dedicated to a study of these functors. 
Keeping an eye towards the main goal of this text, we must avoid assuming that $A$ is noetherian. Hence, we do not know if the completion map $A \rightarrow \hat{A}$ is flat. We overcome this issue by using DG-algebras, which will be assumed to be (graded-) commutative. We refer the reader to [Avramov 1998; Keller 1994; Mac Lane 1963; Yekutieli 2016] for information about DG-algebras and their derived categories. For a DG-algebra $A$, we denote by DGMod $A$ the category of DG-modules over $A$, and by $\mathrm{D}(\mathrm{DGMod} A)$ the derived category over $A$.

We shall need the following well known result from DG-homological algebra:

Proposition 3.1. Let $A \rightarrow B$ be a quasiisomorphism between two commutative $D G$-algebras, and let

$$
\operatorname{Rest}_{B / A}: \mathrm{D}(\operatorname{DGMod} B) \rightarrow \mathrm{D}(\operatorname{DGMod} A)
$$

be the forgetful functor.

(1) There is an isomorphism

$$
1_{\mathrm{D}(\mathrm{DGMod} B)} \cong B \otimes_{A}^{\mathrm{L}} \operatorname{Rest}_{B / A}(-)
$$

of functors $\mathrm{D}(\mathrm{DGMod} B) \rightarrow \mathrm{D}(\mathrm{DGMod} B)$.

(2) There is an isomorphism

$$
1_{\mathrm{D}(\operatorname{DGMod} B)} \cong \mathrm{R}_{\operatorname{Hom}_{A}}\left(B, \operatorname{Rest}_{B / A}(-)\right)
$$

of functors $\mathrm{D}(\mathrm{DGMod} B) \rightarrow \mathrm{D}(\mathrm{DGMod} B)$.

Proof. Part (1) follows immediately from [Stacks 2005-, Tag 09S6], or [Yekutieli 2016, Proposition 2.5(1)], while part (2) follows immediately from [Shaul 2016, Lemma 2.2], or [Yekutieli 2016, Proposition 2.5(2)].

As far as we know, the next results are new even in the case where $A$ is noetherian. In the noetherian case, one does not need DG-algebras in the proof of the next result.

Theorem 3.2. Let $A$ be a commutative ring, let $\mathfrak{a} \subseteq$ A be a finitely generated ideal, and let $\boldsymbol{a}$ be a finite sequence that generates $\mathfrak{a}$. Assume that $\mathfrak{a}$ is weakly proregular. Then there is an isomorphism of functors

$$
\mathrm{R} \hat{\Gamma}_{\mathfrak{a}}(-) \cong \hat{A} \otimes_{A}^{\mathrm{L}}\left(\mathrm{K}_{\infty}^{\vee}(A ; \boldsymbol{a}) \otimes_{A}-\right) .
$$

Proof. Set $\hat{A}:=\Lambda_{\mathfrak{a}}(A)$. Consider the completion map $A \rightarrow \hat{A}$. Since $A$ is not necessarily noetherian, this map might fail to be flat, so let $A \stackrel{f}{\rightarrow} \tilde{A} \stackrel{g}{\rightarrow} \hat{A}$ be a K-flat DG-algebra resolution of $A \rightarrow \hat{A}$. That is, $f: A \rightarrow \tilde{A}$ is a K-flat DG-algebra map, $g: \tilde{A} \rightarrow \hat{A}$ is a quasiisomorphism of DG-algebras, and $g \circ f$ is equal to the completion map $A \rightarrow \hat{A}$. We denote by

$\operatorname{Rest}_{\hat{A} / \tilde{A}}: \mathrm{D}(\operatorname{Mod} \hat{A}) \rightarrow \mathrm{D}(\operatorname{DGMod} \tilde{A}) \quad$ and $\quad \operatorname{Rest}_{\tilde{A} / A}: \mathrm{D}(\operatorname{DGMod} \tilde{A}) \rightarrow \mathrm{D}(\operatorname{Mod} A)$ 
the corresponding forgetful functors. Set

$$
\mathrm{R} \tilde{\Gamma}_{\mathfrak{a}}(-):=\operatorname{Rest}_{\hat{A} / \tilde{A}} \circ \mathrm{R} \hat{\Gamma}_{\mathfrak{a}}(-): \mathrm{D}(\operatorname{Mod} A) \rightarrow \mathrm{D}(\operatorname{DGMod} \tilde{A}) .
$$

Let $M \in \mathrm{D}(\operatorname{Mod} A)$. Let $P \rightarrow M$ be a K-flat resolution of $M$, and let $M \rightarrow I$ be a Kinjective resolution of $M$. The map $f: A \rightarrow \tilde{A}$ induces a map $1_{P} \otimes_{A} f: P \rightarrow P \otimes_{A} \tilde{A}$. Let $P \otimes_{A} \tilde{A} \rightarrow J$ be a K-injective resolution of $P \otimes_{A} \tilde{A}$ over $\tilde{A}$. Because $f$ is flat, $J$ is also a K-injective resolution of $P \otimes_{A} \tilde{A}$ over $A$. There is a unique map $\phi: I \rightarrow J$ in $\mathrm{K}(\operatorname{Mod} A)$, which makes the diagram

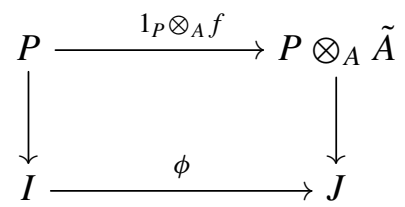

commutative, and it induces a map $\Gamma_{\mathfrak{a}}(\phi): \Gamma_{\mathfrak{a}}(I) \rightarrow \Gamma_{\mathfrak{a}}(J)$. Our goal is to show that $\Gamma_{\mathfrak{a}}(\phi)$ is a quasiisomorphism. The morphism of functors

$$
\alpha(-): \Gamma_{\mathfrak{a}}(-) \rightarrow \mathrm{K}_{\infty}^{\vee}(A ; \boldsymbol{a}) \otimes_{A}-
$$

that was constructed in [Porta et al. 2014b, Equation (4.19)], and the map $\phi$ induce the commutative diagram

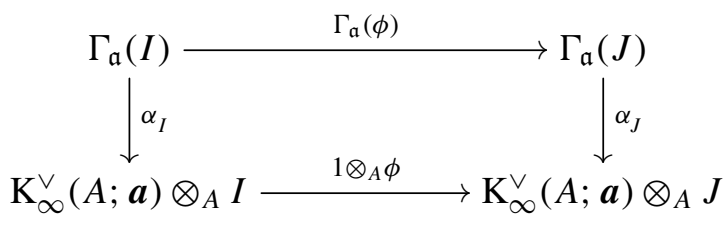

Because $\boldsymbol{a}$ is weakly proregular, the two vertical maps are quasiisomorphisms. We claim that the bottom horizontal map is also a quasiisomorphism. To see this, consider the following commutative diagram in $\mathrm{K}(\operatorname{Mod} A)$ :

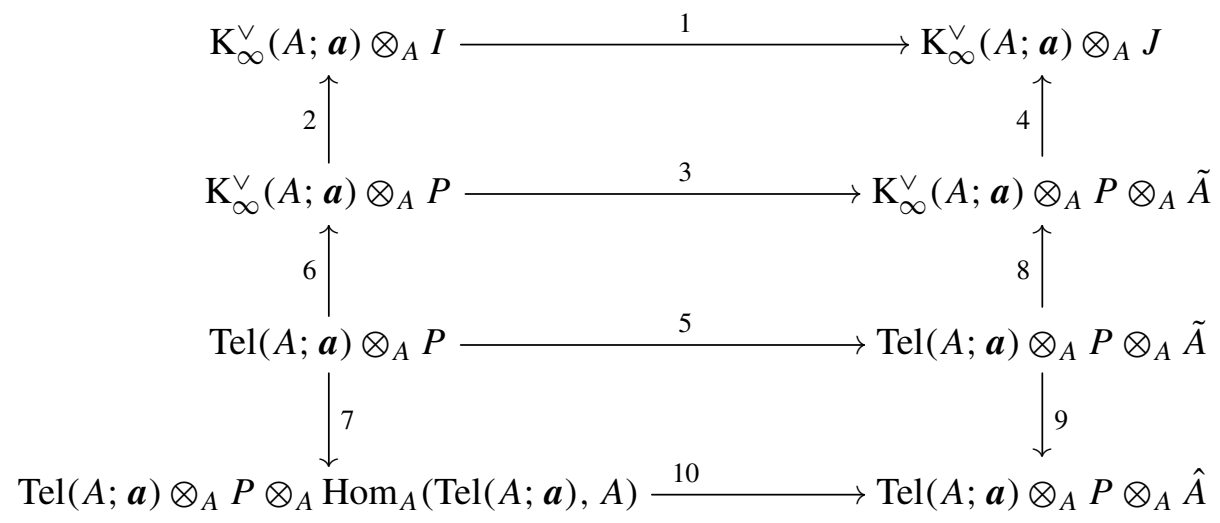


The top square in this diagram is induced from the square (3.3), the middle square is induced from the quasiisomorphism

$$
\operatorname{Tel}(A ; \boldsymbol{a}) \rightarrow \mathrm{K}_{\infty}^{\vee}(A ; \boldsymbol{a}),
$$

and the bottom square is induced from the commutative diagram [Porta et al. 2014b, Equation (5.26)]. By [Porta et al. 2014b, Corollary 5.23], $\operatorname{Hom}_{A}(\operatorname{Tel}(A ; \boldsymbol{a}), A) \rightarrow \hat{A}$ is a quasiisomorphism. Since $\operatorname{Tel}(A ; \boldsymbol{a})$ and $P$ are both K-flat, it follows that (10) is a quasiisomorphism. K-flatness of $P$ also implies that (9) is a quasiisomorphism. The map (7), which is induced by the map $A \rightarrow \operatorname{Hom}_{A}(\operatorname{Tel}(A ; \boldsymbol{a}), A)$ is a quasiisomorphism by [Alonso Tarrío et al. 1997, Corollary after Theorem (0.3)*] (or the proof of [Porta et al. 2014b, Lemma 7.6]). Hence, the map (5) is also a quasiisomorphism. It is clear that (6) and (8) are quasiisomorphisms, so that (3) is also a quasiisomorphism. As (2) and (4) are also quasiisomorphisms, we deduce that (1) is a quasiisomorphism. Returning to the commutative diagram (3.4), we deduce that the map

$$
\Gamma_{\mathfrak{a}}(\phi): \Gamma_{\mathfrak{a}}(I) \rightarrow \Gamma_{\mathfrak{a}}(J)
$$

is a quasiisomorphism.

There are functorial isomorphisms in $\mathrm{D}(\operatorname{DGMod} \tilde{A})$ :

$$
\mathrm{R} \tilde{\Gamma}_{\mathfrak{a}}(M)=\operatorname{Rest}_{\hat{A} / \tilde{A}}\left(\mathrm{R} \hat{\Gamma}_{\mathfrak{a}}(M)\right) \cong \operatorname{Rest}_{\hat{A} / \tilde{A}}\left(\hat{\Gamma}_{\mathfrak{a}}(I)\right) .
$$

Since the map $\Gamma_{\mathfrak{a}}(\phi): \Gamma_{\mathfrak{a}}(I) \rightarrow \Gamma_{\mathfrak{a}}(J)$ is a quasiisomorphism, it follows that

$$
\operatorname{Rest}_{\hat{A} / \tilde{A}}\left(\hat{\Gamma}_{\mathfrak{a}}(\phi)\right): \operatorname{Rest}_{\hat{A} / \tilde{A}}\left(\hat{\Gamma}_{\mathfrak{a}}(I)\right) \rightarrow \operatorname{Rest}_{\hat{A} / \tilde{A}}\left(\hat{\Gamma}_{\mathfrak{a}}(J)\right)
$$

is also a quasiisomorphism. The DG $\tilde{A}$-module $\operatorname{Rest}_{\hat{A} / \tilde{A}}\left(\hat{\Gamma}_{\mathfrak{a}}(J)\right)$ is a sub-DG-module of $J$, and the inclusion map induces a map

$$
\operatorname{Rest}_{\hat{A} / \tilde{A}}\left(\hat{\Gamma}_{\mathfrak{a}}(J)\right) \rightarrow \mathrm{K}_{\infty}^{\vee}(A ; \boldsymbol{a}) \otimes_{A} J .
$$

Applying the forgetful functor $\operatorname{Rest}_{\tilde{A} / A}$ to the map in (3.5) yields the quasiisomorphism

$$
\Gamma_{\mathfrak{a}}(J) \cong \mathrm{K}_{\infty}^{\vee}(A ; \boldsymbol{a}) \otimes_{A} J,
$$

so that the map in (3.5) is also a quasiisomorphism. Hence,

$$
\mathrm{R} \tilde{\Gamma}_{\mathfrak{a}}(M) \cong \mathrm{K}_{\infty}^{\vee}(A ; \boldsymbol{a}) \otimes_{A} J \cong \mathrm{K}_{\infty}^{\vee}(A ; \boldsymbol{a}) \otimes_{A} M \otimes_{A} \tilde{A} .
$$

By Proposition 3.1, there is an isomorphism of functors

$$
1_{\mathrm{D}(\mathrm{DGMod} \hat{A})} \cong \hat{A} \otimes_{\tilde{A}}^{\mathrm{L}} \operatorname{Rest}_{\hat{A} / \tilde{A}}(-) .
$$

Hence,

$$
\mathrm{R} \hat{\Gamma}_{\mathfrak{a}}(-) \cong \hat{A} \otimes_{\tilde{A}}^{\mathrm{L}} \mathrm{R} \tilde{\Gamma}_{\mathfrak{a}}(-),
$$


which implies that

$$
\mathrm{R} \hat{\Gamma}_{\mathfrak{a}}(M) \cong \hat{A} \otimes_{A}^{\mathrm{L}}\left(\mathrm{K}_{\infty}^{\vee}(A ; \boldsymbol{a}) \otimes_{A} M\right) .
$$

Dually, we have the next result for the $\mathrm{L} \hat{\Lambda}_{\mathfrak{a}}$ functor. Note however that, in this case, even if $A$ is noetherian, we have to use DG-algebra resolutions, because $\hat{A}$ is almost never projective over $A$ (see, for example, [Buchweitz and Flenner 2006, Theorem 2.1]).

Theorem 3.6. Let $A$ be a commutative ring, let $\mathfrak{a} \subseteq$ A be a finitely generated ideal, and let $\boldsymbol{a}$ be a finite sequence that generates $\mathfrak{a}$. Assume that $\mathfrak{a}$ is weakly proregular. Then there is an isomorphism of functors

$$
\mathrm{L} \hat{\Lambda}_{\mathfrak{a}}(-) \cong \operatorname{RHom}_{A}\left(\hat{A} \otimes_{A} \operatorname{Tel}(A ; \boldsymbol{a}),-\right) .
$$

Proof. We use notation as in the proof of Theorem 3.2. Let $A \stackrel{f}{\rightarrow} \tilde{A} \stackrel{g}{\rightarrow} \hat{A}$ be a K-projective DG-algebra resolution of $A \rightarrow \hat{A}$, and set

$$
\mathrm{L} \tilde{\Lambda}_{\mathfrak{a}}(-):=\operatorname{Rest}_{\hat{A} / \tilde{A}} \circ \mathrm{L} \hat{\Lambda}_{\mathfrak{a}}(-): \mathrm{D}(\operatorname{Mod} A) \rightarrow \mathrm{D}(\operatorname{DGMod} \tilde{A}) .
$$

Let $M \in \mathrm{D}(\operatorname{Mod} A)$. Let $P \rightarrow M$ be a K-projective resolution, and let $M \rightarrow I$ be a Kinjective resolution. The map $f: A \rightarrow \tilde{A}$ induces a map $\operatorname{Hom}_{A}(f, 1): \operatorname{Hom}_{A}(\tilde{A}, I) \rightarrow I$. Let $Q \rightarrow \operatorname{Hom}_{A}(\tilde{A}, I)$ be a K-projective resolution of $\operatorname{Hom}_{A}(\tilde{A}, I)$ over $\tilde{A}$. There is a unique map $\phi: Q \rightarrow P$ in $\mathrm{K}(\operatorname{Mod} A)$ making the diagram

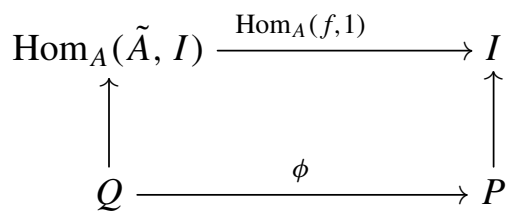

commutative. The morphism of functors

$$
\beta(-): \operatorname{Hom}_{A}(\operatorname{Tel}(A ; \boldsymbol{a}),-) \rightarrow \Lambda_{\mathfrak{a}}(-)
$$

that was constructed in [Porta et al. 2014b, Definition 5.16] and the map $\phi: Q \rightarrow P$ induce a commutative diagram

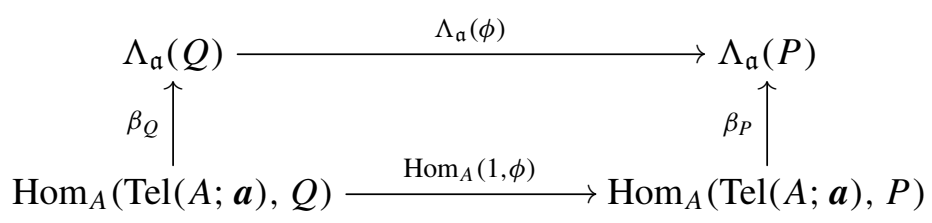

and, because of weak proregularity of $\boldsymbol{a}$, the two vertical maps in this diagram are quasiisomorphisms. We will show that the bottom horizontal map is also a quasiisomorphism, which will imply that the top horizontal map is a quasiisomorphism. 
To see this, consider the commutative diagram

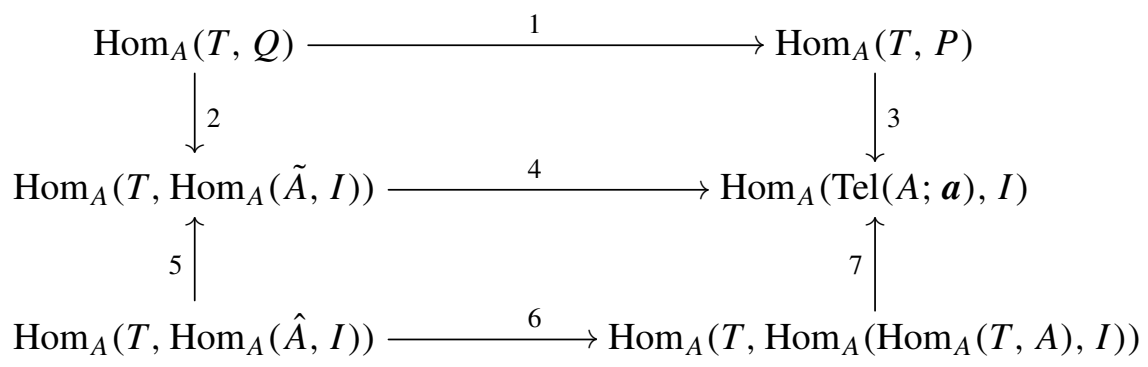

in $\mathrm{K}(\operatorname{Mod} A)$, where we have set $T:=\operatorname{Tel}(A ; \boldsymbol{a})$. The top square of this diagram is induced from the square (3.7), while the bottom square is induced from the commutative diagram of [Porta et al. 2014b, Equation (5.26)]. Weak proregularity of $\boldsymbol{a}$ implies that the map (6) is a quasiisomorphism. The fact that $I$ is K-injective and that $\operatorname{Tel}(A ; \boldsymbol{a})$ is $\mathrm{K}$-projective implies that (5) is a quasiisomorphism. The hom-tensor adjunction and [Alonso Tarrío et al. 1997, Corollary after Theorem (0.3)*] (or the proof of [Porta et al. 2014b, Lemma 7.6]) shows that (7) is also a quasiisomorphism. Hence, (4) is a quasiisomorphism. As (2) and (3) are clearly quasiisomorphisms, we deduce that (1) is a quasiisomorphism. Returning to the commutative diagram (3.8), we deduce that the map

$$
\Lambda_{\mathfrak{a}}(\phi): \Lambda_{\mathfrak{a}}(Q) \rightarrow \Lambda_{\mathfrak{a}}(P)
$$

is a quasiisomorphism.

There are functorial isomorphisms

$$
\mathrm{L} \tilde{\Lambda}_{\mathfrak{a}}(M)=\operatorname{Rest}_{\hat{A} / \tilde{A}}\left(\mathrm{~L} \hat{\Lambda}_{\mathfrak{a}}(M)\right) \cong \operatorname{Rest}_{\hat{A} / \tilde{A}}\left(\hat{\Lambda}_{\mathfrak{a}}(P)\right)
$$

in $\mathrm{D}(\operatorname{DGMod} \tilde{A})$, and since the map $\Lambda_{\mathfrak{a}}(\phi): \Lambda_{\mathfrak{a}}(Q) \rightarrow \Lambda_{\mathfrak{a}}(P)$ is a quasiisomorphism, it follows that the map

$$
\operatorname{Rest}_{\hat{A} / \tilde{A}}\left(\hat{\Lambda}_{\mathfrak{a}}(\phi)\right): \operatorname{Rest}_{\hat{A} / \tilde{A}}\left(\hat{\Lambda}_{\mathfrak{a}}(Q)\right) \rightarrow \operatorname{Rest}_{\hat{A} / \tilde{A}}\left(\hat{\Lambda}_{\mathfrak{a}}(P)\right)
$$

is also a quasiisomorphism. By [Porta et al. 2014b, Corollary 5.23], there is an $A$-linear quasiisomorphism

$$
\beta_{Q}: \operatorname{Hom}_{A}(\operatorname{Tel}(A ; \boldsymbol{a}), Q) \rightarrow \Lambda_{\mathfrak{a}}(Q),
$$

and it is easy to verify that the same construction gives rise to an $\tilde{A}$-linear quasiisomorphism

$$
\operatorname{Hom}_{A}(\operatorname{Tel}(A ; \boldsymbol{a}), Q) \rightarrow \operatorname{Rest}_{\hat{A} / \tilde{A}}\left(\hat{\Lambda}_{\mathfrak{a}}(Q)\right)
$$


Hence, there are isomorphisms of functors

$$
\begin{aligned}
\operatorname{L} \tilde{\Lambda}_{\mathfrak{a}}(M) & \cong \operatorname{Hom}_{A}(\operatorname{Tel}(A ; \boldsymbol{a}), Q) \\
& \cong \operatorname{Hom}_{A}\left(\operatorname{Tel}(A ; \boldsymbol{a}), \operatorname{Hom}_{A}(\tilde{A}, I)\right) \\
& \cong \operatorname{RHom}_{A}\left(\operatorname{Tel}(A ; \boldsymbol{a}) \otimes_{A} \tilde{A}, M\right) .
\end{aligned}
$$

By Proposition 3.1, there is an isomorphism of functors

$$
1_{\mathrm{D}(\operatorname{DGMod} \hat{A})} \cong \operatorname{R}_{\operatorname{Hom}_{\tilde{A}}}\left(\hat{A}, \operatorname{Rest}_{\hat{A} / \tilde{A}}(-)\right) \text {. }
$$

Hence,

$$
\mathrm{L} \hat{\Lambda}_{\mathfrak{a}}(-) \cong \operatorname{RHom}_{\tilde{A}}\left(\hat{A}, \mathrm{~L} \tilde{\Lambda}_{\mathfrak{a}}(-)\right)
$$

which implies that

$$
\mathrm{L} \hat{\Lambda}_{\mathfrak{a}}(M) \cong \mathrm{R} \operatorname{Hom}_{A}\left(\operatorname{Tel}(A ; \boldsymbol{a}) \otimes_{A} \hat{A}, M\right) .
$$

Corollary 3.9. Let $A$ be a commutative ring, let $\mathfrak{a} \subseteq A$ be a weakly proregular ideal, and let $M, N \in \mathrm{D}(\operatorname{Mod} A)$. Then there are isomorphisms

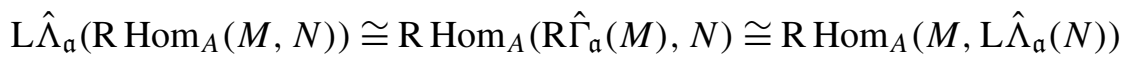

of functors

$$
\mathrm{D}(\operatorname{Mod} A) \times \mathrm{D}(\operatorname{Mod} A) \rightarrow \mathrm{D}(\operatorname{Mod} \hat{A}) .
$$

Proof. Let $\boldsymbol{a}$ be a finite sequence that generates $\mathfrak{a}$. The hom-tensor adjunction and the quasiisomorphism $\mathrm{K}_{\infty}^{\vee}(A ; \boldsymbol{a}) \cong \operatorname{Tel}(A ; \boldsymbol{a})$ show that there are bifunctorial isomorphisms

$\mathrm{R}_{\operatorname{Hom}}\left(\operatorname{Tel}(A ; \boldsymbol{a}) \otimes_{A} \hat{A}, \mathrm{R}_{\operatorname{Hom}_{A}}(M, N)\right) \cong \mathrm{R} \operatorname{Hom}_{A}\left(\mathrm{~K}_{\infty}^{\vee}(A ; \boldsymbol{a}) \otimes_{A} \hat{A} \otimes_{A}^{\mathrm{L}} M, N\right)$ and

$\mathrm{R}_{\operatorname{Hom}}\left(\mathrm{K}_{\infty}^{\vee}(A ; \boldsymbol{a}) \otimes_{A} \hat{A} \otimes_{A}^{\mathrm{L}} M, N\right) \cong \mathrm{R} \operatorname{Hom}_{A}\left(M, \mathrm{R}_{\operatorname{Hom}}\left(\operatorname{Tel}(A ; \boldsymbol{a}) \otimes_{A} \hat{A}, N\right)\right)$, so the result follows from Theorems 3.2 and 3.6.

Remark 3.10. If $A=\hat{A}$, then the above corollary collapses to the Greenlees-May duality (see [Alonso Tarrío et al. 1997, Theorem (0.3)], or [Porta et al. 2014b, Theorem 7.12]).

The next two corollaries will be applied in the next section to study relations between the derived completion functor and Hochschild cohomology.

Corollary 3.11. Let $A$ be a commutative ring, and let $\mathfrak{a} \subseteq A$ be a weakly proregular ideal. Given a ring map $\hat{A} \rightarrow B$, and a complex $M \in \mathrm{D}(\operatorname{Mod} B)$, there is an isomorphism

$$
\mathrm{R}_{\operatorname{Hom}}\left(M, \mathrm{~L} \Lambda_{\mathfrak{a}}(-)\right) \cong \mathrm{R}_{\operatorname{Hom}}\left(M, \mathrm{~L} \hat{\Lambda}_{\mathfrak{a}}(-)\right)
$$


of functors

$$
\mathrm{D}(\operatorname{Mod} A) \rightarrow \mathrm{D}(\operatorname{Mod} B)
$$

Proof. Let $\boldsymbol{a}$ be a finite sequence that generates $\mathfrak{a}$. Let $N \in \mathrm{D}(\operatorname{Mod} A)$. By Theorem 3.6, there is an isomorphism of functors

$$
\operatorname{R}_{\operatorname{Hom}}^{\hat{A}}\left(M, \operatorname{L} \hat{\Lambda}_{\mathfrak{a}}(N)\right) \cong \operatorname{R}_{\operatorname{Hom}_{\hat{A}}}\left(M, \operatorname{R}_{\operatorname{Hom}}\left(\operatorname{Tel}(A ; \boldsymbol{a}) \otimes_{A} \hat{A}, N\right)\right) .
$$

Applying the hom-tensor adjunction twice, we get an isomorphism $\operatorname{R}_{\operatorname{Hom}_{\hat{A}}}\left(M, \operatorname{R}_{\operatorname{Hom}_{A}}\left(\operatorname{Tel}(A ; \boldsymbol{a}) \otimes_{A} \hat{A}, N\right)\right) \cong \operatorname{RHom}_{A}\left(M, \operatorname{Hom}_{A}(\operatorname{Tel}(A ; \boldsymbol{a}), N)\right)$, which proves the claim.

Corollary 3.12. Let $A$ be a commutative ring, and let $B$ be a commutative A-algebra. Let $\mathfrak{a} \subseteq A$ be a weakly proregular ideal, let $\mathfrak{b}=\mathfrak{a} \cdot B$, and assume that $\mathfrak{b}$ is also weakly proregular. Then there is an isomorphism

$$
\mathrm{L} \hat{\Lambda}_{\mathfrak{b}} \mathrm{R} \operatorname{Hom}_{A}(B,-) \cong \mathrm{R} \operatorname{Hom}_{A}\left(\hat{B}, \mathrm{~L} \Lambda_{\mathfrak{a}}(-)\right)
$$

of functors

$$
\mathrm{D}(\operatorname{Mod} A) \rightarrow \mathrm{D}(\operatorname{Mod} \hat{B}),
$$

where $\hat{B}:=\Lambda_{\mathfrak{b}}(B)$.

Proof. Let $\boldsymbol{a}$ be a finite sequence that generates $\mathfrak{a}$, and let $\boldsymbol{b}$ be its image in $B$. By Theorem 3.6, given $M \in \mathrm{D}(\operatorname{Mod} A)$, there is a functorial isomorphism

$$
\mathrm{L}_{\hat{\Lambda}} \mathrm{R}_{\operatorname{Hom}_{A}}(B, M) \cong \mathrm{R} \operatorname{Hom}_{B}\left(\hat{B} \otimes_{B} \operatorname{Tel}(B ; \boldsymbol{b}), \mathrm{R}_{\operatorname{Hom}_{A}}(B, M)\right),
$$

so, by the derived hom-tensor adjunction,

$$
\mathrm{L} \hat{\Lambda}_{\mathfrak{b}} \mathrm{R}_{H^{\prime}}(B, M) \cong \operatorname{Rom}_{A}\left(\hat{B} \otimes_{B} \operatorname{Tel}(B ; \boldsymbol{b}), M\right) .
$$

By the base change property of the telescope complex, we have

$$
\operatorname{Tel}(B ; \boldsymbol{b}) \cong B \otimes_{A} \operatorname{Tel}(A ; \boldsymbol{a}),
$$

so that

$$
\mathrm{L} \hat{\Lambda}_{\mathfrak{b}} \mathrm{R} \operatorname{Hom}_{A}(B, M) \cong \mathrm{R} \operatorname{Hom}_{A}\left(\hat{B} \otimes_{A} \operatorname{Tel}(A ; \boldsymbol{a}), M\right) .
$$

Hence, using adjunction again, and the fact that $\mathfrak{a}$ is weakly proregular, we obtain the result.

Dually to these two corollaries, we have the following results which will apply to the study of Hochschild homology. We omit the very similar proofs. 
Corollary 3.13. Let $A$ be a commutative ring, and let $\mathfrak{a} \subseteq A$ be a weakly proregular ideal. Given a ring map $\hat{A} \rightarrow B$, and a complex $M \in \mathrm{D}(\operatorname{Mod} B)$, there is an isomorphism

$$
M \otimes_{A}^{\mathrm{L}} \mathrm{R} \Gamma_{\mathfrak{a}}(-) \cong M \otimes_{\hat{A}}^{\mathrm{L}} \mathrm{R} \hat{\Gamma}_{\mathfrak{a}}(-)
$$

of functors

$$
\mathrm{D}(\operatorname{Mod} A) \rightarrow \mathrm{D}(\operatorname{Mod} B)
$$

Corollary 3.14. Let $A$ be a commutative ring, and let $B$ be a commutative A-algebra. Let $\mathfrak{a} \subseteq A$ be a weakly proregular ideal, let $\mathfrak{b}=\mathfrak{a} \cdot B$, and assume that $\mathfrak{b}$ is also weakly proregular. Then there is an isomorphism

$$
\left.\mathrm{R} \hat{\Gamma}_{\mathfrak{b}}\left(B \otimes_{A}^{\mathrm{L}}-\right) \cong \hat{B} \otimes_{A}^{\mathrm{L}} \mathrm{R} \Gamma_{\mathfrak{a}}(-)\right)
$$

of functors

$$
\mathrm{D}(\operatorname{Mod} A) \rightarrow \mathrm{D}(\operatorname{Mod} \hat{B}),
$$

where $\hat{B}:=\Lambda_{\mathfrak{b}}(B)$.

\section{Hochschild cohomology and derived completion}

We now turn to the main theme of this paper: relations between adic completion (and its derived functor) and Hochschild cohomology. The results of this section rely heavily on the tools developed in the previous sections.

Our first result describes the effect of applying the derived completion functor to the Hochschild cohomology complex in a rather general situation. We will later specialize further to obtain more explicit results.

Theorem 4.1. Let $\mathbb{k}$ be a commutative ring, let $A$ be a flat noetherian $\mathbb{k}$-algebra, and let $\mathfrak{a} \subseteq A$ be an ideal. Assume further that at least one of the following holds:

(1) The ring $\mathbb{k}$ is an absolutely flat ring (e.g., a field).

(2) The ring $A / \mathfrak{a}$ is essentially of finite type over $\mathbb{k}$.

(3) The ideal $I:=\mathfrak{a} \otimes_{\mathbb{k}} A+A \otimes_{\mathbb{k}} \mathfrak{a} \subseteq A \otimes_{\mathbb{k}} A$ is weakly proregular.

Set $\hat{A}:=\Lambda_{\mathfrak{a}}(A)$ and $A \hat{\otimes}_{\mathbb{k}} A:=\Lambda_{I}\left(A \otimes_{\mathbb{k}} A\right)$. Then there are isomorphisms

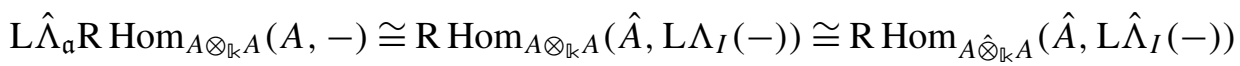

of functors

$$
\mathrm{D}\left(\operatorname{Mod} A \otimes_{\mathbb{k}} A\right) \rightarrow \mathrm{D}(\operatorname{Mod} \hat{A}) .
$$

Proof. If $\mathbb{k}$ is absolutely flat, then $I$ is weakly proregular by Proposition 2.1, while if $A / \mathfrak{a}$ is essentially of finite type over $\mathbb{k}$, then $I$ is weakly proregular by Theorem 2.6. Since the image of $I$ in $A$ is equal to $\mathfrak{a}$, and since $A$ being noetherian implies that 
$\mathfrak{a}$ is weakly proregular, given $M \in \mathrm{D}\left(\operatorname{Mod} A \otimes_{\mathbb{k}} A\right)$, by Corollary 3.12, there is a functorial isomorphism

$$
\mathrm{L} \hat{\Lambda}_{\mathfrak{a}} \mathrm{R} \operatorname{Hom}_{A \otimes_{\mathbb{k}} A}(A, M) \cong \operatorname{RHom}_{A \otimes_{\mathbb{k}} A}\left(\hat{A}, \operatorname{L} \Lambda_{I}(M)\right) .
$$

Note that, considered as an $\left(A \otimes_{\mathbb{k}} A\right)$-module, the $I$-adic completion of $A$ is equal to $\hat{A}$. Hence, we may apply Corollary 3.11 , and deduce that there is a functorial isomorphism

$$
\mathrm{R}_{\operatorname{Hom}}^{A \otimes_{\mathbb{k}} A}\left(\hat{A}, \mathrm{~L} \Lambda_{I}(M)\right) \cong \operatorname{RHom}_{A \hat{\otimes}_{k} A}\left(\hat{A}, \mathrm{~L} \hat{\Lambda}_{I}(M)\right)
$$

in $\mathrm{D}(\operatorname{Mod} \hat{A})$. This proves the result.

The next lemma might seem trivial at first glance. However, the possible lack of flatness of the completion map makes it a little more difficult.

Lemma 4.2. Let $A$ be a commutative ring, and let $\mathfrak{a} \subseteq A$ be a weakly proregular ideal. Assume that $\hat{A}:=\Lambda_{\mathfrak{a}}(A)$ is noetherian, and let $M \in \operatorname{Mod} \hat{A}$ be an $\mathfrak{a}$-adically complete $\hat{A}$-module. Let

$$
\operatorname{Rest}_{\hat{A} / A}: \mathrm{D}(\operatorname{Mod} \hat{A}) \rightarrow \mathrm{D}(\operatorname{Mod} A)
$$

be the forgetful functor. Then there is a functorial isomorphism

$$
\operatorname{L}_{\mathfrak{a}}\left(\operatorname{Rest}_{\hat{A} / A}(M)\right) \cong \operatorname{Rest}_{\hat{A} / A}(M)
$$

in $\mathrm{D}(\operatorname{Mod} A)$, and a functorial isomorphism

$$
\mathrm{L} \hat{\Lambda}_{\mathfrak{a}}\left(\operatorname{Rest}_{\hat{A} / A}(M)\right) \cong M
$$

in $\mathrm{D}(\operatorname{Mod} \hat{A})$.

Proof. Let $\boldsymbol{a}$ be a finite sequence that generates $\mathfrak{a}$, and let $\hat{\boldsymbol{a}}$ be its image in $\hat{A}$. Since $\hat{A}$ is noetherian and $M$ is $\mathfrak{a}$-adically complete, it follows from [Porta et al. 2015, Theorem 1.21] that $M$ is cohomologically $\mathfrak{a} \hat{A}$-adically complete, so there is an isomorphism

$$
M \cong \operatorname{RHom}_{\hat{A}}(\operatorname{Tel}(\hat{A} ; \hat{\boldsymbol{a}}), M) .
$$

The base change property of the telescope complex implies that $\operatorname{Tel}(A ; \boldsymbol{a}) \otimes_{A} \hat{A} \cong$ $\operatorname{Tel}(\hat{A} ; \hat{\boldsymbol{a}})$. Using this fact and the hom-tensor adjunction, we deduce that

so that

$$
\operatorname{Rest}_{\hat{A} / A}(M) \cong \operatorname{RHom}_{A}\left(\operatorname{Tel}(A ; \boldsymbol{a}), \operatorname{Rest}_{\hat{A} / A}(M)\right),
$$

$$
\operatorname{L}_{\mathfrak{a}}\left(\operatorname{Rest}_{\hat{A} / A}(M)\right) \cong \operatorname{Rest}_{\hat{A} / A}(M) .
$$

Note that

$$
\operatorname{Rest}_{\hat{A} / A}\left(\operatorname{L} \hat{\Lambda}_{\mathfrak{a}}\left(\operatorname{Rest}_{\hat{A} / A}(M)\right)\right) \cong \operatorname{L}_{\mathfrak{a}}\left(\operatorname{Rest}_{\hat{A} / A}(M)\right) \cong \operatorname{Rest}_{\hat{A} / A}(M)
$$


Hence, the complex of $\hat{A}$-modules

$$
\operatorname{L} \hat{\Lambda}_{\mathfrak{a}}\left(\operatorname{Rest}_{\hat{A} / A}(M)\right)
$$

is concentrated in degree 0 . Letting $P \rightarrow \operatorname{Rest}_{\hat{A} / A}(M)$ be a projective resolution, we deduce that the map

$$
\hat{\Lambda}_{\mathfrak{a}}(P) \rightarrow \hat{\Lambda}_{\mathfrak{a}}\left(\operatorname{Rest}_{\hat{A} / A}(M)\right) \cong M
$$

is an $\hat{A}$-linear quasiisomorphism, so the result follows from the fact that

$$
\operatorname{L} \hat{\Lambda}_{\mathfrak{a}}\left(\operatorname{Rest}_{\hat{A} / A}(M)\right) \cong \hat{\Lambda}_{\mathfrak{a}}(P) .
$$

Using this lemma, and as a first corollary of Theorem 4.1, we obtain the next result which reduces the problem of computing the Hochschild cohomology of an adically complete ring to a problem over noetherian rings.

Corollary 4.3. Let $\mathbb{k}$ be a commutative ring, and let $A$ be a flat noetherian $\mathbb{k}$-algebra . Assume $\mathfrak{a} \subseteq A$ is an ideal such that $A$ is a-adically complete, and such that $A / \mathfrak{a}$ is essentially of finite type over $\mathbb{k}$. Let $I=\mathfrak{a} \otimes_{\mathbb{k}} A+A \otimes_{\mathbb{k}} \mathfrak{a}$, and set $A \hat{\otimes}_{\mathbb{k}} A:=\Lambda_{I}\left(A \otimes_{\mathbb{k}} A\right)$. Then for any $M \in \operatorname{Mod} A \otimes_{\mathbb{k}} A$ which is I-adically complete (for example, any a-adically complete A-module, or, more particularly, any finitely generated A-module), there is a functorial isomorphism

$$
\mathrm{R}_{H^{\prime}} \operatorname{Qom}_{A \mathbb{k}} A(A, M) \cong \operatorname{RHom}_{A \hat{\otimes}_{\mathbb{k}} A}(A, M)
$$

in $\mathrm{D}(\operatorname{Mod} A)$, and the ring $A \hat{\otimes}_{\mathbb{k}} A$ is noetherian.

Proof. That $A \hat{\otimes}_{\mathbb{k}} A$ is noetherian follows from the fact that $\left(A \otimes_{\mathbb{k}} A\right) / I \cong A / \mathfrak{a} \otimes_{\mathbb{k}} A / \mathfrak{a}$ is noetherian, and $I$ is finitely generated. Since $A=\hat{A}$, according to Theorem 4.1, there is a functorial isomorphism

$$
\mathrm{R}_{\operatorname{Hom}}^{A \otimes_{\mathbb{k}} A}\left(A, \mathrm{~L} \Lambda_{I}(M)\right) \cong \mathrm{R}_{\operatorname{Hom}}^{A \hat{\otimes}_{k} A}\left(A, \mathrm{~L} \hat{\Lambda}_{I}(M)\right) .
$$

By Lemma 4.2, we have

$$
\mathrm{L} \Lambda_{I}(M) \cong M \quad \text { in } \mathrm{D}\left(\operatorname{Mod} A \otimes_{\mathbb{k}} A\right)
$$

and

$$
\mathrm{L} \hat{\Lambda}_{I}(M) \cong M \quad \text { in } \mathrm{D}\left(\operatorname{Mod} A \hat{\otimes}_{\mathbb{k}} A\right) .
$$

Using these two facts, we obtain the functorial isomorphism

$$
\mathrm{R} \operatorname{Hom}_{A \otimes_{\mathbb{k}} A}(A, M) \cong \mathrm{R} \operatorname{Hom}_{A \hat{\otimes}_{\mathbb{k}} A}(A, M) .
$$

Remark 4.4. In [Buchweitz and Flenner 2006, Section 3, page 113], the authors defined the analytic Hochschild cohomology and discussed its relation to ordinary Hochschild cohomology. The setup there is as follows: $\left(A, \mathfrak{m}_{A}\right)$ and $\left(B, \mathfrak{m}_{B}\right)$ are two complete noetherian local rings, and there is a flat local map $A \rightarrow B$ such that 
the induced map $A / \mathfrak{m}_{A} \rightarrow B / \mathfrak{m}_{B}$ is an isomorphism. In this situation, the authors defined the analytic bar resolution $\hat{\mathcal{B}}$ by replacing $B^{\otimes_{A}^{n}}$ with its completion with respect to the maximal ideal $\operatorname{ker}\left(B^{\otimes_{A}^{n}} \rightarrow B / \mathfrak{m}_{B}\right)$ in the ordinary bar resolution. Using this complex, the authors defined the analytic Hochschild cohomology by

$$
\widehat{\mathrm{HH}}^{n}(B / A, M):=H^{n} \operatorname{Hom}_{B \hat{\otimes}_{A} B}(\hat{\mathcal{B}}, M),
$$

observed that there is a canonical map

$$
\widehat{\mathrm{HH}}^{n}(B / A, M) \rightarrow \mathrm{HH}^{n}(B / A, M),
$$

and asked if these modules are isomorphic. Using Corollary 4.3 and the results of [Buchweitz and Flenner 2006], we may now obtain a positive answer to this question:

Corollary 4.5. Let $\left(A, \mathfrak{m}_{A}\right) \rightarrow\left(B, \mathfrak{m}_{B}\right)$ be a flat local homomorphism of complete noetherian local rings such that the induced map of residue fields $A / \mathfrak{m}_{A} \rightarrow B / \mathfrak{m}_{B}$ is an isomorphism. Then for any $\mathfrak{m}_{B}$-adically complete B-module $M$ (in particular, for any finitely generated $B$-module $M$ ), there is a natural B-module isomorphism

$$
\widehat{\mathrm{HH}}^{n}(B / A, M) \cong \mathrm{HH}^{n}(B / A, M) .
$$

Proof. Note that $M$ is $\operatorname{ker}\left(B \otimes_{A} B \rightarrow B / \mathfrak{m}_{B}\right)$-adically complete, so by [Buchweitz and Flenner 2006, Proposition 3.1], the natural map

$$
\operatorname{HH}^{n}(B / A, M) \rightarrow \operatorname{Ext}_{B \otimes_{A} B}^{n}(B, M)
$$

is an isomorphism. Similarly, by [Buchweitz and Flenner 2006, Proposition 3.2], the natural map

$$
\widehat{\mathrm{HH}}^{n}(B / A, M) \rightarrow \operatorname{Ext}_{B \hat{\otimes}_{A} B}^{n}(B, M)
$$

is an isomorphism. Finally, by Corollary 4.3, there is a natural isomorphism

$$
\operatorname{Ext}_{B \otimes_{A} B}^{n}(B, M) \cong \operatorname{Ext}_{B \hat{\otimes}_{A} B}^{n}(B, M),
$$

so the result follows.

Our next goal is to give the complex appearing in Theorem 4.1 a description in terms of the Hochschild complex of the ring $\hat{A}$. First we need a lemma.

Lemma 4.6. Let $\mathbb{k}$ be a commutative ring, let $A$ be a flat noetherian $\mathbb{k}$-algebra, and let $\mathfrak{a} \subseteq A$ be an ideal. Let

$$
I:=\mathfrak{a} \otimes_{\mathbb{k}} A+A \otimes_{\mathbb{k}} \mathfrak{a} \subseteq A \otimes_{\mathbb{k}} A,
$$

let $\hat{\mathfrak{a}}:=\mathfrak{a} \cdot \Lambda_{\mathfrak{a}}(A)$, and let

$$
J=\hat{\mathfrak{a}} \otimes_{\mathbb{k}} \hat{A}+\hat{A} \otimes_{\mathbb{k}} \hat{\mathfrak{a}}=I \cdot\left(\hat{A} \otimes_{\mathbb{k}} \hat{A}\right) \subseteq \hat{A} \otimes_{\mathbb{k}} \hat{A} .
$$


If $\tau_{A}: A \rightarrow \hat{A}$ is the completion map, then the ring map

$$
\Lambda_{I}\left(\tau_{A} \otimes_{k} \tau_{A}\right): \Lambda_{I}\left(A \otimes_{\mathbb{k}} A\right) \rightarrow \Lambda_{J}\left(\hat{A} \otimes_{\mathbb{k}} \hat{A}\right)
$$

is an isomorphism.

Proof. Notice that for each $n$ we have

$$
I^{n}=\sum_{i=0}^{n} \mathfrak{a}^{i} \otimes \mathfrak{a}^{n-i},
$$

where we have set $\mathfrak{a}^{0}=A$. Hence,

$$
\mathfrak{a}^{2 n} \otimes_{\mathbb{k}} A+A \otimes_{\mathbb{k}} \mathfrak{a}^{2 n} \subseteq I^{2 n} \subseteq \mathfrak{a}^{n} \otimes_{\mathbb{k}} A+A \otimes_{\mathbb{k}} \mathfrak{a}^{n} .
$$

It follows that the two sequences of ideals $\left\{I^{n}\right\}$ and $\left\{\mathfrak{a}^{n} \otimes_{\mathbb{k}} A+A \otimes_{\mathbb{k}} \mathfrak{a}^{n}\right\}$ are cofinal in each other, so that

$$
\lim _{(}\left(A \otimes_{\mathbb{k}} A\right) / I^{n} \cong \lim \left(A \otimes_{\mathbb{k}} A\right) /\left(\mathfrak{a}^{n} \otimes_{\mathbb{k}} A+A \otimes_{\mathbb{k}} \mathfrak{a}^{n}\right) \cong \lim A / \mathfrak{a}^{n} \otimes_{\mathbb{k}} A / \mathfrak{a}^{n} .
$$

Since $A$ is noetherian, $\hat{A}$ is flat over $A$, so it is also flat over $\mathbb{k}$. Hence, in the exact same manner,

$$
\lim _{(}\left(\hat{A} \otimes_{\mathbb{k}} \hat{A}\right) / J^{n} \cong \lim \hat{A} /(\hat{\mathfrak{a}})^{n} \otimes_{\mathbb{k}} A /(\hat{\mathfrak{a}})^{n} .
$$

Hence, the result follows from the fact that $\hat{A} /(\hat{\mathfrak{a}})^{n} \cong A / \mathfrak{a}^{n}$, and from the observation that the maps $\Lambda_{I}\left(\tau_{A} \otimes_{k} \tau_{A}\right)$ and

$$
\underset{\lim }{\longleftarrow}\left(\left(\tau_{A} \otimes_{k} \tau_{A}\right) /\left(\mathfrak{a}^{n} \otimes_{\mathbb{k}} A+A \otimes_{\mathbb{k}} \mathfrak{a}^{n}\right)\right)
$$

are equal.

Remark 4.7. In the notation of the above lemma, note that composing the completion map

$$
\hat{A} \otimes_{\mathbb{k}} \hat{A} \rightarrow \Lambda_{J}\left(\hat{A} \otimes_{\mathbb{k}} \hat{A}\right)
$$

with the isomorphism

$$
\Lambda_{J}\left(\hat{A} \otimes_{\mathbb{k}} \hat{A}\right) \rightarrow \Lambda_{I}\left(A \otimes_{\mathbb{k}} A\right)
$$

we obtain a ring map

$$
\hat{A} \otimes_{\mathbb{k}} \hat{A} \rightarrow \Lambda_{I}\left(A \otimes_{\mathbb{k}} A\right) .
$$

Using this map, given $M \in \operatorname{Mod}\left(A \otimes_{\mathbb{k}} A\right)$, we will be able to regard

$$
\hat{\Lambda}_{I}(M) \in \operatorname{Mod}\left(\Lambda_{I}\left(A \otimes_{\mathbb{k}} A\right)\right)
$$

as an $\left(\hat{A} \otimes_{\mathbb{k}} \hat{A}\right)$-module which is $J$-adically complete. 
We may now refine Theorem 4.1, and present the derived completion of the Hochschild cohomology complex as a Hochschild cohomology complex over the completion:

Theorem 4.8. Let $\mathbb{k}$ be a commutative ring, and let $A$ be a flat noetherian $\mathbb{k}$-algebra such that $A \otimes_{\mathbb{k}} A$ is noetherian. Let $\mathfrak{a} \subseteq A$ be an ideal, and let $M$ be a finitely generated $\left(A \otimes_{\mathbb{k}} A\right)$-module. Set

$$
I:=\mathfrak{a} \otimes_{\mathbb{k}} A+A \otimes_{\mathbb{k}} \mathfrak{a} \subseteq A \otimes_{\mathbb{k}} A .
$$

Then there is a functorial isomorphism

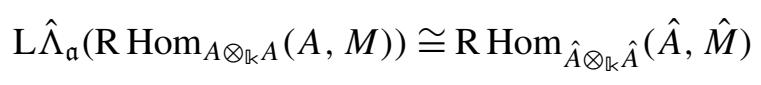

in $\mathrm{D}(\operatorname{Mod} \hat{A})$, where $\hat{A}:=\hat{\Lambda}_{\mathfrak{a}}(A)$ and $\hat{M}:=\hat{\Lambda}_{I}(M)$.

Proof. Set $A \hat{\otimes}_{\mathbb{k}} A:=\Lambda_{I}\left(A \otimes_{\mathbb{k}} A\right)$. By assumption, $A \otimes_{\mathbb{k}} A$ is noetherian, so $I$ is weakly proregular. According to Theorem 4.1, there is a functorial isomorphism

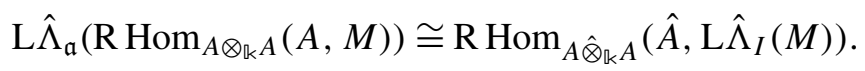

Because $A \otimes_{\mathbb{k}} A$ is noetherian and $M$ is a finitely generated module, it follows that

$$
\mathrm{L} \hat{\Lambda}_{I}(M) \cong \hat{\Lambda}_{I}(M)=\hat{M}
$$

in $\mathrm{D}\left(\operatorname{Mod} A \hat{\otimes}_{\mathbb{k}} A\right)$. Hence, by Lemma 4.6, there is a functorial isomorphism

$$
\mathrm{R}^{\operatorname{Hom}_{A \hat{\otimes}_{k} A}}(\hat{A}, \hat{M}) \cong \operatorname{RHom}_{\hat{A} \hat{\otimes}_{k} \hat{A}}(\hat{A}, \hat{M})
$$

in $\mathrm{D}(\operatorname{Mod} \hat{A})$. Let $\hat{\mathfrak{a}}:=\mathfrak{a} \cdot \hat{A} \subseteq \hat{A}$. Since $A$ is noetherian, the map $A \rightarrow \hat{A}$ is flat, so the map $A \otimes_{\mathbb{k}} A \rightarrow \hat{A} \otimes_{\mathbb{k}} \hat{A}$ is also flat. Hence, by Remark 2.2, the ideal $J:=I \cdot\left(\hat{A} \otimes_{\mathbb{k}} \hat{A}\right)=\hat{\mathfrak{a}} \otimes_{\mathbb{k}} \hat{A}+\hat{A} \otimes_{\mathbb{k}} \hat{\mathfrak{a}}$ is weakly proregular.

Because $A \otimes_{\mathbb{k}} A$ is noetherian, its completion $\Lambda_{I}\left(A \otimes_{\mathbb{k}} A\right)$ is also noetherian, so, again by Lemma 4.6 , the $\operatorname{ring} \Lambda_{J}\left(\hat{A} \otimes_{\mathbb{k}} \hat{A}\right)$ is noetherian. Hence, by Lemma 4.2, $\mathrm{L} \Lambda_{J}(\hat{M}) \cong \mathrm{L} \hat{\Lambda}_{J}(\hat{M}) \cong \hat{M}$. Applying Theorem 4.1 to the ring $\hat{A}$, we have a functorial isomorphism

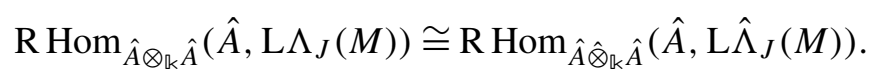

Composing this isomorphism with the isomorphisms of (4.9) and (4.10), we obtain the result.

Our final goal in this section is to show that when taking cohomology in the above theorem, derived completion may be replaced with ordinary completion. The next simple lemma is needed for the proof of Proposition 4.12. 
Lemma 4.11. Let $A$ be a noetherian ring, let $P$ be a bounded complex of free $A$-modules, and let $M$ be a finitely generated A-module. Then the canonical map

$$
\operatorname{Hom}_{A}(P, A) \otimes_{A} M \rightarrow \operatorname{Hom}_{A}(P, M)
$$

is an isomorphism of complexes.

Proof. It is enough to show this in the case where $P$ is a single free $A$-module. In that case, note that $\operatorname{Hom}_{A}(P, A)$ is a direct product of copies of $A$, and since $A$ is noetherian this is a flat $A$-module. Thus, both of the functors $\operatorname{Hom}_{A}(P, A) \otimes_{A}-$ and $\operatorname{Hom}_{A}(P,-)$ are exact, and if $M$ is a finitely generated free $A$-module, then the canonical map $\operatorname{Hom}_{A}(P, A) \otimes_{A} M \rightarrow \operatorname{Hom}_{A}(P, M)$ is obviously an isomorphism. Hence, the result of the lemma follows from the standard finite presentation trick.

In the case where $M$ is bounded above, the next proposition is [Frankild 2003, Proposition 2.7]. We, however, wish to apply this result to the Hochschild complex which is bounded below, so we give a proof that works for complexes without any boundedness condition.

Proposition 4.12. Let $A$ be a noetherian ring, and let $\mathfrak{a} \subseteq A$ be an ideal. Then there is an isomorphism

$$
\mathrm{L} \Lambda_{\mathfrak{a}}(M) \cong \hat{A} \otimes_{A} M
$$

of functors

$$
\mathrm{D}_{\mathrm{f}}(\operatorname{Mod} A) \rightarrow \mathrm{D}(\operatorname{Mod} A) .
$$

Proof. There is a sequence of morphisms of functors

$$
\hat{A} \otimes_{A} M \cong \operatorname{Hom}_{A}(\operatorname{Tel}(A ; \boldsymbol{a}), A) \otimes_{A} M \rightarrow \operatorname{Hom}_{A}(\operatorname{Tel}(A ; \boldsymbol{a}), M) \cong \operatorname{L} \Lambda_{\mathfrak{a}}(M) .
$$

Because $A$ is assumed to be noetherian, $\hat{A}$ is flat over $A$. Hence, both $\operatorname{L} \Lambda_{\mathfrak{a}}(-)$ and $-\otimes_{A} \hat{A}$ are functors of finite cohomological dimension. Thus, by [Hartshorne 1966, Proposition I.7.1], it is enough to show that the above morphism is an isomorphism in the case where $M$ is a finitely generated $A$-module, and this follows from Lemma 4.11.

Here is the result promised in the title of this paper:

Theorem 4.13. Let $\mathbb{k}$ be a commutative ring, and let $A$ be a flat noetherian $\mathbb{k}$-algebra such that $A \otimes_{\mathbb{k}} A$ is noetherian. Let $\mathfrak{a} \subseteq A$ be an ideal, and let $M$ be a finitely generated $\left(A \otimes_{\mathbb{k}} A\right)$-module. Then for any $n \in \mathbb{N}$, there is a functorial isomorphism

$$
\Lambda_{\mathfrak{a}}\left(\operatorname{Ext}_{A \otimes_{\mathbb{k}} A}^{n}(A, M)\right) \cong \operatorname{Ext}_{\hat{A} \otimes_{\mathbb{k}} \hat{A}}^{n}(\hat{A}, \hat{M}) .
$$

If , moreover, either

(1) $\mathbb{k}$ is a field, or 
(2) A is projective over $\mathbb{k}$, a is a maximal ideal, and $M$ is a finitely generated A-module,

then there is also a functorial isomorphism

$$
\Lambda_{\mathfrak{a}}\left(\mathrm{HH}^{n}(A / \mathbb{k}, M)\right) \cong \mathrm{HH}^{n}(\hat{A} / \mathbb{k}, \hat{M}) .
$$

Proof. The assumptions of the theorem ensure that

$$
\mathrm{R} \operatorname{Hom}_{A \otimes_{\mathbb{k}} A}(A, M) \in \mathrm{D}_{\mathrm{f}}(\operatorname{Mod} A),
$$

so since $A$ is noetherian, we have a functorial isomorphism

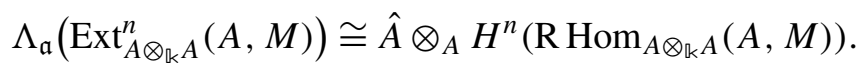

Flatness of $\hat{A}$ over $A$ implies (for example, by [Porta et al. 2014b, Corollary 2.12]) that there is a natural isomorphism

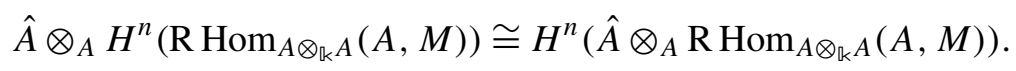

Hence, by Proposition 4.12, it is enough to compute the $n$-th cohomology of the complex

$$
\mathrm{L} \Lambda_{\mathfrak{a}}\left(\mathrm{R} \operatorname{Hom}_{A \otimes_{\mathbb{k}} A}(A, M)\right)
$$

Letting

$$
\operatorname{Rest}_{\hat{A} / A}: \mathrm{D}(\operatorname{Mod} \hat{A}) \rightarrow \mathrm{D}(\operatorname{Mod} A)
$$

be the forgetful functor, the above complex is equal to

$$
\operatorname{Rest}_{\hat{A} / A} \circ \operatorname{L} \hat{\Lambda}_{\mathfrak{a}}\left(\operatorname{RHom}_{A \otimes_{\mathbb{k}} A}(A, M)\right) \text {. }
$$

By Theorem 4.8, there is a functorial isomorphism

$$
\mathrm{L} \hat{\Lambda}_{\mathfrak{a}} \mathrm{R} \operatorname{Hom}_{A \otimes_{\mathbb{k}} A}(A, M) \cong \mathrm{R}_{\operatorname{Hom}_{\hat{A} \otimes_{\mathfrak{k}} \hat{A}}}(\hat{A}, \hat{M})
$$

in $\mathrm{D}(\operatorname{Mod} \hat{A})$, so applying the forgetful functor we obtain an $A$-linear natural isomorphism

$$
\Lambda_{\mathfrak{a}}\left(\operatorname{Ext}_{A \otimes_{\mathbb{k}} A}^{n}(A, M)\right) \cong \operatorname{Ext}_{\hat{A} \otimes_{\mathbb{k}} \hat{A}}^{n}(\hat{A}, \hat{M}) .
$$

(Actually, any $A$-linear map between $\hat{A}$-modules is automatically $\hat{A}$-linear, so this isomorphism is even an isomorphism of $\hat{A}$-modules.) This establishes the first claim of the theorem. If $\mathbb{k}$ is a field then the second claim obviously follows from the first one. Assume now that $A$ is projective over $\mathbb{k}$, that $\mathfrak{a}$ is a maximal ideal, and that $M$ is a finitely generated $A$-module. Let $\phi$ be the composition of the diagonal map $\hat{A} \otimes_{\mathbb{k}} \hat{A} \rightarrow \hat{A}$ with the map $\hat{A} \rightarrow \hat{A} / \mathfrak{a} \hat{A}$. Then $m=\operatorname{ker}(\phi) \subseteq \hat{A} \otimes_{\mathbb{k}} \hat{A}$ is a maximal 
ideal, and the image of $m$ in $\hat{A}$ is equal to $\mathfrak{a} \hat{A}$. Hence $\hat{M}$ is $m$-adically complete, so by [Buchweitz and Flenner 2006, Proposition 3.1] the canonical map

$$
\operatorname{HH}^{n}(\hat{A} / \mathbb{k}, \hat{M}) \rightarrow \operatorname{Ext}_{\hat{A} \otimes_{k} \hat{A}}^{n}(\hat{A}, \hat{M})
$$

is an isomorphism. This proves the second claim.

The above result allows us to compute the Hochschild cohomology of power series rings, which is new as far as we know.

Example 4.14. Let $\mathbb{k}$ be a noetherian ring. Let $A=\mathbb{k}\left[x_{1}, \ldots, x_{n}\right], \mathfrak{a}=\left(x_{1}, \ldots, x_{n}\right)$, and $M=A$. Note that $A$ is projective over $\mathbb{k}$, and that $\Lambda_{\mathfrak{a}}(A)=\mathbb{k} \llbracket x_{1}, \ldots, x_{n} \rrbracket$. Hence, by the above theorem, we have

$$
\operatorname{Ext}_{\hat{A} \otimes_{\mathbb{k}} \hat{A}}^{i}(\hat{A}, \hat{A}) \cong \Lambda_{\mathfrak{a}}\left(\mathrm{HH}^{i}\left(\mathbb{k}\left[x_{1}, \ldots, x_{n}\right] / \mathbb{k}, \mathbb{k}\left[x_{1}, \ldots, x_{n}\right]\right)\right) .
$$

By the Hochschild-Kostant-Rosenberg theorem, the right-hand side is equal to

$$
\Lambda_{\mathfrak{a}} \wedge^{i}\left(\mathbb{k}\left[x_{1}, \ldots, x_{n}\right]^{n}\right),
$$

so we have an isomorphism

$$
\operatorname{Ext}_{\hat{A} \otimes_{\mathbb{k}} \hat{A}}^{i}(\hat{A}, \hat{A}) \cong \wedge^{i}\left(\mathbb{k} \llbracket x_{1}, \ldots, x_{n} \rrbracket^{n}\right) .
$$

If, moreover, $\mathbb{k}$ is a field, we obtain that

$$
\mathrm{HH}^{i}\left(\mathbb{k} \llbracket x_{1}, \ldots, x_{n} \rrbracket / \mathbb{k}, \mathbb{k} \llbracket x_{1}, \ldots, x_{n} \rrbracket\right) \cong \wedge^{i}\left(\mathbb{k} \llbracket x_{1}, \ldots, x_{n} \rrbracket^{n}\right) .
$$

We remark that one can also use Corollary 4.3 to make this calculation.

Example 4.15. Let $A$ be a noetherian ring, and let $\mathfrak{a} \subseteq A$ be an ideal. Then, by Theorem 4.8, we have

$$
\mathrm{R} \operatorname{Hom}_{\hat{A} \otimes_{A} \hat{A}}(\hat{A}, \hat{A}) \cong \mathrm{L}_{\mathfrak{a}} \mathrm{R} \operatorname{Hom}_{A}(A, A)=\hat{A} .
$$

Assume now that $\mathfrak{a}$ is a maximal ideal. Then, by Theorem 4.13,

$$
\operatorname{HH}^{n}(\hat{A} / A, \hat{A})=0
$$

for all $n \neq 0$, and $\operatorname{HH}^{0}(\hat{A} / A, \hat{A})=\hat{A}$. Specializing to the case where $A=\mathbb{Z}$, and $\mathfrak{a}=(p)$ for some prime number $p$, we have computed the absolute Hochschild cohomology of the ring of $p$-adic integers $\mathbb{Z}_{p}$.

Remark 4.16. The above examples are both particular cases of an adic HochschildKostant-Rosenberg theorem which applies for any formally smooth adic algebra $A$ such that $A / \mathfrak{a}$ is essentially of finite type over $\mathbb{k}$. This can be shown using Corollary 4.3 and by studying the local structure of the completed diagonal $\operatorname{ker}\left(A \hat{\otimes}_{\mathbb{k}} A \rightarrow A\right)$. A full proof of this will appear elsewhere. 


\section{Hochschild homology and derived torsion}

In this short and final section we discuss relations between Hochschild homology and the derived torsion functor.

Theorem 5.1. Let $\mathbb{k}$ be a commutative ring, let $A$ be a flat noetherian $\mathbb{k}$-algebra, and let $\mathfrak{a} \subseteq A$ be an ideal. Assume further that at least one of the following holds:

(1) The ring $\mathbb{k}$ is an absolutely flat ring (e.g., a field).

(2) $A / \mathfrak{a}$ is essentially of finite type over $\mathbb{k}$.

(3) The ideal $I:=\mathfrak{a} \otimes_{\mathbb{k}} A+A \otimes_{\mathbb{k}} \mathfrak{a} \subseteq A \otimes_{\mathbb{k}} A$ is weakly proregular.

Set $\hat{A}:=\Lambda_{\mathfrak{a}}(A)$ and $A \hat{\otimes}_{\mathbb{k}} A:=\Lambda_{I}\left(A \otimes_{\mathbb{k}} A\right)$. Then there are isomorphisms

$$
\mathrm{R} \hat{\Gamma}_{\mathfrak{a}}\left(A \otimes_{A \otimes_{\mathbb{k}} A}^{\mathrm{L}}-\right) \cong \hat{A} \otimes_{A \otimes_{\mathbb{k}} A}^{\mathrm{L}} \mathrm{R} \Gamma_{I}(-) \cong \hat{A} \otimes_{A \hat{\otimes}_{\mathfrak{k}} A}^{\mathrm{L}} \mathrm{R} \hat{\Gamma}_{I}(-)
$$

of functors

$$
\mathrm{D}\left(\operatorname{Mod} A \otimes_{\mathbb{k}} A\right) \rightarrow \mathrm{D}(\operatorname{Mod} \hat{A}) .
$$

Proof. As in the proof of Theorem 4.1, the first two conditions imply the third one, so we may assume $I$ is weakly proregular. The first isomorphism then follows from Corollary 3.14, while the second isomorphism follows from Corollary 3.13.

Remark 5.2. In view of Theorem 4.13, it is natural to ask if Hochschild homology also commutes with adic completion. Here, the answer is false, even in simple situations. Indeed, let $\mathbb{k}$ be a field of characteristic 0 , let $A=\mathbb{k}[x]$, and let $\mathfrak{a}=(x)$. Then

$$
\mathrm{HH}_{1}(A / \mathbb{k}, A) \cong A,
$$

so that $\Lambda_{\mathfrak{a}}\left(\mathrm{HH}_{1}(A / \mathbb{k}, A)\right) \cong \mathbb{k} \llbracket x \rrbracket$. On the other hand, $\mathrm{HH}_{1}(\hat{A} / \mathbb{k}, \hat{A}) \cong \Omega_{\mathbb{k} \llbracket x \rrbracket / \mathbb{k}}^{1}$ is an infinitely generated $\mathbb{k} \llbracket x \rrbracket$-module.

Remark 5.3. As an alternative to the badly behaved Hochschild homology of commutative adic algebras, Hübl [1989] developed a theory of adic Hochschild homology by studying the cohomologies of the functor

$$
A \otimes_{A \hat{\otimes}_{\mathbb{k}} A}^{\mathrm{L}}-\text {. }
$$

As our Corollary 4.3 shows, in the case of Hochschild cohomology, usual Hochschild cohomology coincides with adic Hochschild cohomology, but by the previous remark we see that for Hochschild homology this is not the case.

\section{Acknowledgments}

The author would like to thank Amnon Yekutieli for some helpful suggestions. The author is grateful for the anonymous referees for their helpful comments. 


\section{References}

[Alonso Tarrío et al. 1997] L. Alonso Tarrío, A. Jeremías López, and J. Lipman, "Local homology and cohomology on schemes”, Ann. Sci. École Norm. Sup. (4) 30:1 (1997), 1-39. MR 1422312 Zbl 0894.14002

[Alonso Tarrío et al. 1999] L. Alonso Tarrío, A. Jeremías López, and J. Lipman, Duality and flat base change on formal schemes, Contemporary Mathematics 244, American Mathematical Society, Providence, RI, 1999. Correction at Proc. Amer. Math. Soc. 131:2 (2003), 351-357. MR 1716706 Zbl 0927.00024

[Avramov 1998] L. L. Avramov, "Infinite free resolutions", pp. 1-118 in Six lectures on commutative algebra (Bellaterra, 1996), edited by J. Elias et al., Progress in Mathematics 166, Birkhäuser, Basel, 1998. MR 1648664 Zbl 0934.13008

[Braunling et al. 2015] O. Braunling, M. Groechenig, and J. Wolfson, "A generalized Contou-Carrère symbol and its reciprocity laws in higher dimensions", preprint, 2015. arXiv 1410.3451v2

[Brodmann and Sharp 2013] M. P. Brodmann and R. Y. Sharp, Local cohomology: an algebraic introduction with geometric applications, 2nd ed., Cambridge Studies in Advanced Mathematics 136, Cambridge University Press, 2013. MR 3014449 Zbl 1263.13014

[Buchweitz and Flenner 2006] R.-O. Buchweitz and H. Flenner, "Power series rings and projectivity", Manuscripta Math. 119:1 (2006), 107-114. MR 2194381 Zbl 1084.13005

[Cartan and Eilenberg 1956] H. Cartan and S. Eilenberg, Homological algebra, Princeton University Press, 1956. MR 0077480 Zbl 0075.24305

[Frankild 2003] A. Frankild, "Vanishing of local homology", Math. Z. 244:3 (2003), 615-630. MR 1992028 Zbl 1020.13003

[Greenlees and May 1992] J. P. C. Greenlees and J. P. May, "Derived functors of $I$-adic completion and local homology”, J. Algebra 149:2 (1992), 438-453. MR 1172439 Zbl 0774.18007

[Grothendieck and Dieudonne 1960] A. Grothendieck and J. Dieudonne, "Éléments de géométrie algébrique, I: Le langage des schémas", Inst. Hautes Études Sci. Publ. Math. 4 (1960), 5-214. MR 0217083 Zb1 0118.36206

[Hartshorne 1966] R. Hartshorne, Residues and duality, Lecture Notes in Mathematics 20, Springer, Berlin, 1966. MR 0222093 Zbl 0212.26101

[Hartshorne 1977] R. Hartshorne, Algebraic geometry, Graduate Texts in Mathematics 52, Springer, New York, 1977. MR 0463157 Zbl 0367.14001

[Hochschild 1945] G. Hochschild, "On the cohomology groups of an associative algebra", Ann. of Math. (2) 46 (1945), 58-67. MR 0011076 Zbl 0063.02029

[Hübl 1989] R. Hübl, Traces of differential forms and Hochschild homology, Lecture Notes in Mathematics 1368, Springer, Berlin, 1989. MR 995670 Zbl 0675.13019

[Ionescu 2001] C. Ionescu, "Hochschild (co)homology in commutative algebra: a survey", An. Ştiinţ. Univ. Ovidius Constanţa Ser. Mat. 9:2 (2001), 87-96. MR 2075931 Zbl 1090.13502

[Keller 1994] B. Keller, "Deriving DG categories", Ann. Sci. École Norm. Sup. (4) 27:1 (1994), 63-102. MR 1258406 Zbl 0799.18007

[Loday 1998] J.-L. Loday, Cyclic homology, 2nd ed., Grundlehren der Mathematischen Wissenschaften 301, Springer, Berlin, 1998. MR 1600246 Zbl 0885.18007

[Lurie 2011] J. Lurie, "Derived algebraic geometry, XII: Proper morphisms, completions, and the Grothendieck existence theorem", preprint, 2011, http://www.math.harvard.edu/ lurie/papers/ DAG-XII.pdf. 
[Mac Lane 1963] S. Mac Lane, Homology, Grundlehren der Mathematischen Wissenschaften 114, Springer, Berlin, 1963. Reprinted in Classics in Mathematics, 1995. MR 0156879 Zbl 0133.26502

[Porta et al. 2014a] M. Porta, L. Shaul, and A. Yekutieli, "Completion by derived double centralizer", Algebr. Represent. Theory 17:2 (2014), 481-494. MR 3181733 Zbl 06306474

[Porta et al. 2014b] M. Porta, L. Shaul, and A. Yekutieli, "On the homology of completion and torsion”, Algebr. Represent. Theory 17:1 (2014), 31-67. MR 3160712 Zbl 06306453

[Porta et al. 2015] M. Porta, L. Shaul, and A. Yekutieli, "Cohomologically cofinite complexes", Comm. Algebra 43:2 (2015), 597-615. MR 3274024 Zbl 06422500

[Schenzel 2003] P. Schenzel, "Proregular sequences, local cohomology, and completion", Math. Scand. 92:2 (2003), 161-180. MR 1973941 Zbl 1023.13011

[Shaul 2016] L. Shaul, "Tensor product of dualizing complexes over a field", preprint, 2016. To appear in J. Commut. Algebra. arXiv 1412.3759v2

[Simon 1990] A.-M. Simon, "Some homological properties of complete modules", Math. Proc. Cambridge Philos. Soc. 108:2 (1990), 231-246. MR 1074711 Zbl 0719.13007

[Spaltenstein 1988] N. Spaltenstein, "Resolutions of unbounded complexes", Compositio Math. 65:2 (1988), 121-154. MR 932640 Zbl 0636.18006

[Stacks 2005-] “The Stacks Project”, electronic reference, 2005-, http://stacks.math.columbia.edu.

[Weibel 1994] C. A. Weibel, An introduction to homological algebra, Cambridge Studies in Advanced Mathematics 38, Cambridge University Press, 1994. MR 1269324 Zbl 0797.18001

[Yekutieli 2011] A. Yekutieli, "On flatness and completion for infinitely generated modules over Noetherian rings", Comm. Algebra 39:11 (2011), 4221-4245. MR 2855123 Zbl 1263.13028

[Yekutieli 2016] A. Yekutieli, "The squaring operation for commutative DG rings", J. Algebra 449 (2016), 50-107. MR 3448166

Communicated by Hubert Flenner

Received 2015-05-23 Revised 2016-03-04 Accepted 2016-05-16

Liran.Shaul@uantwerpen.be Departement Wiskunde-Informatica, Universiteit Antwerpen, Middelheim Campus, Middelheimlaan 1, 2020 Antwerp, Belgium 


\section{Algebra \& Number Theory}

msp.org/ant

\section{EDITORS}

MANAGING EDITOR

Bjorn Poonen

Massachusetts Institute of Technology

Cambridge, USA

\author{
EDITORIAL BOARD CHAIR \\ David Eisenbud \\ University of California \\ Berkeley, USA
}

BOARD OF EDITORS

$\begin{aligned} \text { Dave Benson } & \text { University of Aberdeen, Scotland } & \text { Susan Montgomery } & \text { University of Southern California, USA } \\ \text { Richard E. Borcherds } & \text { University of California, Berkeley, USA } & \text { Shigefumi Mori } & \text { RIMS, Kyoto University, Japan } \\ \text { John H. Coates } & \text { University of Cambridge, UK } & \text { Raman Parimala } & \text { Emory University, USA } \\ \text { J-L. Colliot-Thélène } & \text { CNRS, Université Paris-Sud, France } & \text { Jonathan Pila } & \text { University of Oxford, UK } \\ \text { Brian D. Conrad } & \text { Stanford University, USA } & \text { Anand Pillay } & \text { University of Notre Dame, USA } \\ \text { Hélène Esnault } & \text { Freie Universität Berlin, Germany } & \text { Victor Reiner } & \text { University of Minnesota, USA } \\ \text { Hubert Flenner } & \text { Ruhr-Universität, Germany } & \text { Peter Sarnak } & \text { Princeton University, USA } \\ \text { Sergey Fomin } & \text { University of Michigan, USA } & \text { Joseph H. Silverman } & \text { Brown University, USA } \\ \text { Edward Frenkel } & \text { University of California, Berkeley, USA } & \text { Michael Singer } & \text { North Carolina State University, USA } \\ \text { Andrew Granville } & \text { Université de Montréal, Canada } & \text { Vasudevan Srinivas } & \text { Tata Inst. of Fund. Research, India } \\ \text { Joseph Gubeladze } & \text { San Francisco State University, USA } & \text { J. Toby Stafford } & \text { University of Michigan, USA } \\ \text { Roger Heath-Brown } & \text { Oxford University, UK } & \text { Ravi Vakil } & \text { Stanford University, USA } \\ \text { Craig Huneke } & \text { University of Virginia, USA } & \text { Michel van den Bergh } & \text { Hasselt University, Belgium } \\ \text { Kiran S. Kedlaya } & \text { Univ. of California, San Diego, USA } & \text { Marie-France Vignéras } & \text { Université Paris VII, France } \\ \text { János Kollár } & \text { Princeton University, USA } & \text { Kei-Ichi Watanabe } & \text { Nihon University, Japan } \\ \text { Yuri Manin } & \text { Northwestern University, USA } & \text { Efim Zelmanov } & \text { University of California, San Diego, USA } \\ \text { Philippe Michel } & \text { École Polytechnique Fédérale de Lausanne } & \text { Shou-Wu Zhang } & \text { Princeton University, USA }\end{aligned}$

PRODUCTION

production@msp.org

Silvio Levy, Scientific Editor

See inside back cover or msp.org/ant for submission instructions.

The subscription price for 2016 is US $\$ 290$ /year for the electronic version, and $\$ 485 /$ year (+\$55, if shipping outside the US) for print and electronic. Subscriptions, requests for back issues and changes of subscribers address should be sent to MSP.

Algebra \& Number Theory (ISSN 1944-7833 electronic, 1937-0652 printed) at Mathematical Sciences Publishers, 798 Evans Hall \#3840, c/o University of California, Berkeley, CA 94720-3840 is published continuously online. Periodical rate postage paid at Berkeley, CA 94704, and additional mailing offices.

ANT peer review and production are managed by EditFLow ${ }^{\circledR}$ from MSP.

\section{PUBLISHED BY}

- mathematical sciences publishers

nonprofit scientific publishing

http://msp.org/

() 2016 Mathematical Sciences Publishers 


\section{Algebra \& Number Theory}

Volume $10 \quad$ No. $5 \quad 2016$

Conjugacy classes of special automorphisms of the affine spaces

JÉRÉMY BLANC

Inversion of adjunction for rational and Du Bois pairs

SÁNDOR J. KOVÁCS and KARL SCHWEDE

Hochschild cohomology commutes with adic completion

LIRAN SHAUL

Bifurcations, intersections, and heights

LAURA DEMARCO

RANKEyA DATTA and KaREN E. SMITH

1091

Hoffmann's conjecture for totally singular forms of prime degree

STEPHEN SCULLY 